\title{
Stochastic Optimal Tracking with Preview for Linear Discrete Time Markovian Jump Systems
}

\author{
Gou Nakura \\ 56-2-402, Gokasyo-Hirano, Uji, Kyoto, 611-0011, \\ Japan
}

\section{Introduction}

It is well known that, for the design of tracking control systems, preview information of reference signals is very useful for improving performance of the systems, and recently much work has been done for preview control systems [Cohen \& Shaked (1997); Gershon et al. (2004a); Gershon et al. (2004b); Nakura (2008a); Nakura (2008b); Nakura (2008c); Nakura (2008d); Nakura (2008e); Nakura (2009); Nakura (2010); Sawada (2008); Shaked \& Souza (1995); Takaba (2000)]. Especially, in order to design tracking control systems for a class of systems with rapid or abrupt changes, it is effective in improving the tracking performance to construct tracking control systems considering future information of reference signals. Shaked et al. have constructed the $\mathrm{H} \infty$ tracking control theory with preview for continuousand discrete-time linear time-varying systems by a game theoretic approach [Cohen \& Shaked (1997); Shaked \& Souza (1995)]. Recently the author has extended their theory to linear impulsive systems [Nakura (2008b); Nakura (2008c)]. It is also very important to consider the effects of stochastic noise or uncertainties for tracking control systems. By Gershon et al., the theory of stochastic $\mathrm{H} \infty$ tracking with preview has been presented for linear continuous- and discrete-time systems [Gershon et al. (2004a); Gershon et al. (2004b)]. The Ho tracking theory by the game theoretic approach can be restricted to the optimal or stochastic optimal tracking theory and also extended to the stochastic $\mathrm{H} \infty$ tracking control theory. While some command generators of reference signals are needed in the papers [Sawada (2008); Takaba (2000)], a priori knowledge of any dynamic models for reference signals is not assumed on the game theoretic approach. Also notice that all these works have been studied for the systems with no mode transitions, i.e., the single mode systems. Tracking problems with preview for systems with some mode transitions are also very important issues to research.

Markovian jump systems [Boukas (2006); Costa \& Tuesta (2003); Costa et al. (2005); Dragan \& Morozan (2004); Fragoso (1989); Fragoso (1995); Lee \& Khargonekar (2008); Mariton (1990); Souza \& Fragoso (1993); Sworder (1969); Sworder (1972)] have abrupt random mode changes in their dynamics. The mode changes follow Markov processes. Such systems may be found in the area of mechanical systems, power systems, manufacturing systems, communications, aerospace systems, financial engineering and so on. Such systems are classified into continuous-time [Boukas (2006); Dragan \& Morozan (2004); Mariton (1990); 
Souza \& Fragoso (1993); Sworder (1969); Sworder (1972)] and discrete-time [Costa \& Tuesta (2003); Costa et al. (2005); Lee \& Khargonekar (2008); Fragoso (1989); Fragoso et al. (1995)] systems. The optimal, stochastic optimal and $\mathrm{H} \infty$ control theory has been presented for each of these systems respectively [Costa \& Tuesta (2003); Fragoso (1989); Fragoso et al. (1995); Souza \& Fragoso (1993); Sworder (1969); Sworder (1972)]. The stochastic LQ and Hळ control theory for Markovian jump systems are of high practice. For example, these theories are applied to the solar energy system, the underactuated manipulator system and so on [Costa et al. (2005)]. Although preview compensation for hybrid systems including the Markovian jump systems is very effective for improving the system performance, the preview tracking theory for the Markovian jump systems had not been yet constructed. Recently the author has presented the stochastic LQ and $\mathrm{H}_{\infty}$ preview tracking theories by state feedback for linear continuous-time Markovian jump systems [Nakura (2008d) Nakura (2008e); Nakura (2009)], which are the first theories of the preview tracking control for the Markovian jump systems. For the discrete-time Markovian jump systems, he has presented the stochastic LQ preview tracking theory only by state feedback [Nakura (2010)]. The stochastic LQ preview tracking problem for them by output feedback has not been yet fully investigated.

In this paper we study the stochastic optimal tracking problems with preview by state feedback and output feedback for linear discrete-time Markovian jump systems on the finite time interval and derive the forms of the preview compensator dynamics. In this paper it is assumed that the modes are fully observable in the whole time interval. We consider three different tracking problems according to the structures of preview information and give the control strategies for them respectively. The output feedback dynamic controller is given by using solutions of two types of coupled Riccati difference equations. Feedback controller gains are designed by using one type of coupled Riccati difference equations with terminal conditions, which give the necessary and sufficient conditions for the solvability of the stochastic optimal tracking problem with preview by state feedback, and filter gains are designed by using another type of coupled Riccati difference equations with initial conditions. Correspondingly compensators introducing future information are coupled with each other. This is our very important point in this paper. Finally we consider numerical examples and verify the effectiveness of the preview tracking theory presented in this paper. The organization of this paper is as follows: In section 2 we describe the systems and problem formulation. In section 3 we present the solution of the stochastic optimal preview tracking problems over the finite time interval by state feedback. In section 4 we consider the output feedback problems. In section 5 we consider numerical examples and verify the effectiveness of the stochastic optimal preview tracking design theory. In the appendices we present the proof of the proposition, which gives the necessary and sufficient conditions of the solvability for the stochastic optimal preview tracking problems by state feedback, and the orthogonal property of the variable of the error system and that of the output feedback controller, which plays the important role to solve the output feedback problems.

Notations: Throughout this paper the superscript ' stands for the matrix transposition, $|\cdot|$ denotes the Euclidean vector norm and $|\mathrm{v}|_{R}^{2}$ also denotes the weighted norm $v^{\prime} R v$. O denotes the matrix with all zero components.

\section{Problem formulation}

Let $(\Omega, F, P)$ be a probability space and, on this space, consider the following linear discretetime time-varying system with reference signal and Markovian mode transitions. 


$$
\begin{aligned}
& x(k+1)=A_{d, m(k)}(k) x(k)+G_{d, m(k)}(k) \omega_{d}(k)+B_{2 d, m(k)}(k) u_{d}(k)+B_{3 d, m(k)}(k) r_{d}(k) \\
& \mathrm{z}_{\mathrm{d}}(\mathrm{k})=\mathrm{C}_{1 \mathrm{~d}, \mathrm{~m}(\mathrm{k})}(\mathrm{k}) \mathrm{x}(\mathrm{k})+\mathrm{D}_{12 \mathrm{~d}, \mathrm{~m}(\mathrm{k})}(\mathrm{k}) \mathrm{u}_{\mathrm{d}}(\mathrm{k})+\mathrm{D}_{13 \mathrm{~d}, \mathrm{~m}(\mathrm{k})}(\mathrm{k}) \mathrm{r}_{\mathrm{d}}(\mathrm{k}) \\
& \mathrm{y}(\mathrm{k})=\mathrm{C}_{2 \mathrm{~d}, \mathrm{~m}(\mathrm{k})}(\mathrm{k}) \mathrm{x}(\mathrm{k})+\mathrm{H}_{\mathrm{d}, \mathrm{m}(\mathrm{k})}(\mathrm{k}) \omega_{\mathrm{d}}(\mathrm{k}) \\
& \mathrm{x}(0)=\mathrm{x}_{0}, \mathrm{~m}(0)=\mathrm{i}_{0}
\end{aligned}
$$

where $\mathrm{x} \in R^{n}$ is the state, $\omega_{\mathrm{d}} \in R^{p d}$ is the exogenous random noise, $\mathrm{u}_{\mathrm{d}} \in R^{m}$ is the control input, $\mathrm{z}_{\mathrm{d}} \in R^{k d}$ is the controlled output, $\mathrm{r}_{\mathrm{d}}(\cdot) \in R^{r d}$ is known or measurable reference signal and $\mathrm{y} \in R^{k}$ is the measured output. $\mathrm{x}_{0}$ is an unknown initial state and $\mathrm{i}_{0}$ is a given initial mode.

Let $M$ be an integer and $\{\mathrm{m}(\mathrm{k})\}$ is a Markov process taking values on the finite set $\varphi=\{1,2, \cdots, \mathrm{M}\}$ with the following transition probabilities:

$$
P\{m(k+1)=j \mid m(k)=i\}:=p_{d, i j}(k)
$$

where $p_{d, i j}(k) \geq 0$ is also the transition rate at the jump instant from the mode $i$ to $j, i \neq j$, and $\sum_{j=1}^{M} p_{d, i j}(k)=1$. Let $P_{\mathrm{d}}(\mathrm{k})=\left[\mathrm{p}_{\mathrm{d}, \mathrm{ij}}(\mathrm{k})\right]$ be the transition probability matrix. We assume that all these matrices are of compatible dimensions. Throughout this paper the dependence of the matrices on $\mathrm{k}$ will be omitted for the sake of notational simplicity.

For this system (1), we assume the following conditions:

A1: $\mathrm{D}_{12 \mathrm{~d}, \mathrm{~m}(\mathrm{k})}(\mathrm{k})$ is of full column rank.

A2: $\left.\left.\mathrm{D}_{12 \mathrm{~d}, \mathrm{~m}(\mathrm{k})}\right)^{\prime}(\mathrm{k}) \mathrm{C}_{1 \mathrm{~d}, \mathrm{~m}(\mathrm{k})}(\mathrm{k})=\mathrm{O}, \mathrm{D}_{12 \mathrm{~d}, \mathrm{~m}(\mathrm{k})}\right)^{\prime}(\mathrm{k}) \mathrm{D}_{13 \mathrm{~d}, \mathrm{~m}(\mathrm{k})}(\mathrm{k})=\mathrm{O}$

A3: $E\{x(0)\}=\mu_{0}, E\left\{\omega_{d}(k)\right\}=0$,

$\mathrm{E}\left\{\omega_{\mathrm{d}}(\mathrm{k}) \omega_{\mathrm{d}} \mathrm{d}^{\prime}(\mathrm{k}) 1_{\{\mathrm{m}(\mathrm{k})=\mathrm{i}\}}\right\}=X_{\mathrm{i}}$,

$\mathrm{E}\left\{\mathrm{x}(0) \mathrm{x}^{\prime}(0) 1_{\left\{\mathrm{m}(0)=i_{0}\right\}}\right\}=Q_{i_{0}}(0)$,

$\mathrm{E}\left\{\omega_{\mathrm{d}}(0) \mathrm{x}^{\prime}(0) 1_{\left\{\mathrm{m}(0)=i_{0}\right\}}\right\}=\mathrm{O}$,

$\mathrm{E}\left\{\omega_{\mathrm{d}}(\mathrm{k}) \mathrm{x}^{\prime}(\mathrm{k}) 1_{\{\mathrm{m}(\mathrm{k})=\mathrm{i}\}}\right\}=\mathrm{O}$,

$\mathrm{E}\left\{\omega_{\mathrm{d}}(\mathrm{k}) \mathrm{u}_{\mathrm{d}^{\prime}}(\mathrm{k}) 1_{\{\mathrm{m}(\mathrm{k})=\mathrm{i}\}}\right\}=\mathrm{O}$,

$\mathrm{E}\left\{\omega_{\mathrm{d}}(\mathrm{k}) \mathrm{r}_{\mathrm{d}}{ }^{\prime}(\mathrm{k}) 1_{\{\mathrm{m}(\mathrm{k})=\mathrm{i}\}}\right\}=\mathrm{O}$

where $E$ is the expectation with respect to $m(k)$, and the indicator function $1_{\{m(k)=i\}}:=1$ if $\mathrm{m}(\mathrm{k})=\mathrm{i}$, and $1_{\{\mathrm{m}(\mathrm{k})=\mathrm{i}\}}:=0$ if $\mathrm{m}(\mathrm{k}) \neq \mathrm{i}$.

The stochastic optimal tracking problems we address in this section for the system (1) are to design control laws $\mathrm{u}_{\mathrm{d}}(\cdot) \in \mathrm{l}_{2}[0, \mathrm{~N}-1]$ over the finite horizon $[0, \mathrm{~N}]$, using the information available on the known part of the reference signal $r_{d}(\cdot)$ and minimizing the sum of the energy of $z_{d}(k)$, for the given initial mode $i_{0}$ and the given distribution of $x_{0}$. Considering the stochastic mode transitions and the average of the performance indices over the statistical information of the unknown part of $r_{d}$, we define the following performance index.

$$
\begin{aligned}
\mathrm{J}_{\mathrm{dN}}\left(\mathrm{x}_{0}, \mathrm{u}_{\mathrm{d}}, \mathrm{r}_{\mathrm{d}}\right):=\mathrm{E}\left\{\sum_{k=0}^{N} E_{\overline{R_{k}}}\left\{\left|\mathrm{C}_{1 \mathrm{~d}, \mathrm{~m}(\mathrm{k})}(\mathrm{k}) \mathrm{x}(\mathrm{k})+\mathrm{D}_{13 \mathrm{~d}, \mathrm{~m}(\mathrm{k})}(\mathrm{k}) \mathrm{r}_{\mathrm{d}}(\mathrm{k})\right|^{2}\right\}\right. \\
\\
\left.+\sum_{k=0}^{N-1} E_{\overline{R_{k}}}\left\{\left|\mathrm{D}_{12 \mathrm{~d}, \mathrm{~m}(\mathrm{k})}(\mathrm{k}) \mathrm{u}_{\mathrm{d}}(\mathrm{k})\right|^{2}\right\}\right\}
\end{aligned}
$$

$E_{\overline{R_{k}}}$ means the expectation over $\bar{R}_{k+h}, \mathrm{~h}$ is the preview length of $\mathrm{r}_{\mathrm{d}}(\mathrm{k})$, and $\bar{R}_{k}$ denotes the future information on $\mathrm{r}_{\mathrm{d}}$ at the current time $\mathrm{k}$, i.e., $\bar{R}_{k}:=\left\{\mathrm{r}_{\mathrm{d}}(\mathrm{l}) ; \mathrm{k}<\mathrm{l} \leq \mathrm{N}\right\}$. This introduction of 
$E_{\overline{R_{k}}}$ means that the unknown part of the reference signal follows a stochastic process, whose distribution is allowed to be unknown.

Now we formulate the following optimal fixed-preview tracking problems for the system (1) and the performance index (2). In these problems, it is assumed that, at the current time $k$, $r_{d}(l)$ is known for $l \leq \min (N, k+h)$, where $h$ is the preview length.

The Stochastic Optimal Fixed-Preview Tracking Problem by State Feedback:

Consider the system (1) and the performance index (2), and assume the conditions A1, A2 and A3. Then, find $u_{d}^{*}$ minimizing the performance index (2) where the control strategy $u_{d}^{*}$ (k), $0 \leq \mathrm{k} \leq \mathrm{N}-1$, is based on the information $\mathrm{R}_{\mathrm{k}+\mathrm{h}}:=\left\{\mathrm{r}_{\mathrm{d}}(\mathrm{l}) ; 0 \leq \mathrm{l} \leq \mathrm{k}+\mathrm{h}\right\}$ with $0 \leq \mathrm{h} \leq \mathrm{N}$ and the state information $X_{k}:=\{x(l) ; 0 \leq 1 \leq k\}$.

The Stochastic Optimal Fixed-Preview Tracking Problem by Output Feedback:

Consider the system (1) and the performance index (2), and assume the conditions A1, A2 and A3. Then, find $u_{d}^{*}$ minimizing the performance index (2) where the control strategy $u_{d}^{*}$ (k), $0 \leq \mathrm{k} \leq \mathrm{N}-1$, is based on the information $\mathrm{R}_{\mathrm{k}+\mathrm{h}}:=\left\{\mathrm{r}_{\mathrm{d}}(\mathrm{l}) ; 0 \leq \mathrm{l} \leq \mathrm{k}+\mathrm{h}\right\}$ with $0 \leq \mathrm{h} \leq \mathrm{N}$ and the observed information $\mathrm{Y}_{\mathrm{k}}:=\{\mathrm{y}(\mathrm{l}) ; 0 \leq 1 \leq \mathrm{k}\}$.

Notice that, on these problems, at the current time $k$ to decide the control strategies, $R_{k+h}$ can include any noncausal information in the meaning of that it is allowed that the future information of the reference signals $\left\{r_{d}(l) ; k \leq 1 \leq k+h\right\}$ is inputted to the feedback controllers.

\section{Design of tracking controllers by state feedback}

In this section we consider the state feedback problems.

Now we consider the coupled Riccati difference equations [Costa et al. (2005); Fragoso (1989)]

$$
X_{i}(k)=A_{d, i}{ }^{\prime}(k) E_{i}(X(k+1), k) A_{d, i}(k)+C_{1 d, i}{ }^{\prime} C_{1 d, i}-F_{2, i}{ }^{\prime} T_{2, i} F_{2, i}(k), k=0,1, \ldots
$$

where $\mathrm{E}_{\mathrm{i}}(\mathrm{X}(\mathrm{k}+1), \mathrm{k})=\sum_{j=1}^{M} p_{d, i j}(k) \mathrm{X}_{\mathrm{j}+1}(\mathrm{k}+1), \mathrm{X}(\mathrm{k})=\left(\mathrm{X}_{1}(\mathrm{k}), \cdots, \mathrm{X}_{\mathrm{M}}(\mathrm{k})\right)$,

$$
\begin{aligned}
& \mathrm{T}_{2, \mathrm{i}}(\mathrm{k})=\mathrm{D}_{12 \mathrm{~d}, \mathrm{i}}{ }^{\prime} \mathrm{D}_{12 \mathrm{~d}, \mathrm{i}}+\mathrm{B}_{2 \mathrm{~d}, \mathrm{i}}{ }^{\prime} \mathrm{E}_{\mathrm{i}}(\mathrm{X}(\mathrm{k}+1), \mathrm{k}) \mathrm{B}_{2 \mathrm{~d}, \mathrm{i}}, \\
& \mathrm{R}_{2, \mathrm{i}}(\mathrm{k})=\mathrm{B}_{2 \mathrm{~d}, \mathrm{i}} \mathrm{E}_{\mathrm{i}}(\mathrm{X}(\mathrm{k}+1), \mathrm{k}) \mathrm{A}_{\mathrm{d}, \mathrm{i}}, \\
& \mathrm{F}_{2, \mathrm{i}}(\mathrm{k})=-\mathrm{T}_{2, i}^{-1} R_{2, i}(\mathrm{k})
\end{aligned}
$$

and the following scalar coupled difference equations.

$$
\alpha_{i}(\mathrm{k})=\mathrm{E}_{\mathrm{i}}(\alpha(\mathrm{k}+1), \mathrm{k})+\operatorname{tr}\left\{\mathrm{G}_{\mathrm{d}, \mathrm{i}} \mathrm{X}_{\mathrm{i}} \mathrm{G}_{\mathrm{d}, \mathrm{i}}{ }^{\prime} \mathrm{E}_{\mathrm{i}}(\mathrm{X}(\mathrm{k}+1), \mathrm{k})\right\}
$$

where $\mathrm{E}_{\mathrm{i}}(\alpha(\mathrm{k}+1), \mathrm{k})=\sum_{j=1}^{M} p_{d, i j}(k), \alpha_{j}(\mathrm{k}+1)$ and $\alpha(\mathrm{k})=\left(\alpha_{1}(\mathrm{k}), \ldots, \alpha_{M}(\mathrm{k})\right)$.

Remark 3.1 Note that these coupled Riccati difference equations (3) are the same as those for the standard stochastic linear quadratic (LQ) optimization problem of linear discrete-time Markovian jump systems without considering any exogeneous reference signals nor any preview information [Costa et al. (2005); Fragoso (1989)]. Also notice that the form of the equation (4) is different from [Costa et al. (2005); Fragoso (1989)] in the points that the solution $\alpha(\cdot)$ does not depend on any modes in [Costa et al. (2005)] and the noise matrix $\mathrm{G}_{\mathrm{d}}$ does not depend on any modes in [Fragoso (1989)]. 
We obtain the following necessary and sufficient conditions for the solvability of the stochastic optimal fixed-preview tracking problem by state feedback and an optimal control strategy for it.

Theorem 3.1 Consider the system (1) and the performance index (2). Suppose A1, A2 and A3. Then the Stochastic Optimal Fixed-Preview Tracking Problem by State Feedback for (1) and (2) is solvable if and only if there exist matrices $X_{i}(k) \geq 0$ and scalar functions $\alpha_{i}(\mathrm{k}), \mathrm{i}=1$, $\ldots, \mathrm{M}$, satisfying the conditions $\mathrm{X}_{\mathrm{i}}(\mathrm{N})=\mathrm{C}_{1 \mathrm{~d}, \mathrm{i}}{ }^{\prime}(\mathrm{N}) \mathrm{C}_{1 \mathrm{~d}, \mathrm{i}}(\mathrm{N})$ and $\alpha_{i}(\mathrm{~N})=0$ such that the coupled Riccati equations (3) and the coupled scalar equations (4) hold over [0,N]. Moreover an optimal control strategy for the tracking problem (1) and (2) is given by

$$
u_{d}^{*}(\mathrm{k})=\mathrm{F}_{2, \mathrm{i}}(\mathrm{k}) \mathrm{x}(\mathrm{k})+\mathrm{D}_{\mathrm{u}, \mathrm{i}}(\mathrm{k}) \mathrm{r}_{\mathrm{d}}(\mathrm{k})+\mathrm{D}_{\theta \mathrm{u}, \mathrm{i}}(\mathrm{k}) \mathrm{E}_{\mathrm{i}}\left(\theta_{c}(\mathrm{k}+1), \mathrm{k}\right) \text { for } \mathrm{i}=1, \cdots, \mathrm{M}
$$

where $D_{u, i}(k)=-T_{2, i}^{-1}(k) B_{2 d, i} E_{i}(X(k+1), k) B_{3 d, i} \quad$ and $\quad D_{\theta u, i}(k)=-T_{2, i}^{-1}(k) B_{2 d, i}{ }^{\prime} . \quad \theta_{i}(k), \quad i=1, \quad \cdots, M$, $\mathrm{k} \in[0, \mathrm{~N}]$ satisfies

$$
\begin{aligned}
& \theta_{i}(\mathrm{k})=\bar{A}_{d, i}{ }^{\prime}(\mathrm{k}) \mathrm{E}_{\mathrm{i}}(\theta(\mathrm{k}+1), \mathrm{k})+\bar{B}_{d, i}(\mathrm{k}) \mathrm{r}_{\mathrm{d}}(\mathrm{k}), \\
& \theta_{i}(\mathrm{~N})=\mathrm{C}_{1 \mathrm{~d}, \mathrm{i}}{ }^{\prime} \mathrm{D}_{13 \mathrm{~d}, \mathrm{i}} \mathrm{r}_{\mathrm{d}}(\mathrm{N})
\end{aligned}
$$

where $\mathrm{E}_{\mathrm{i}}(\theta(\mathrm{k}+1), \mathrm{k})=\sum_{j=1}^{M} p_{d, i j}(k) \theta_{j}(\mathrm{k}+1)$ and $\theta(\mathrm{k})=\left(\theta_{1}(\mathrm{k}), \cdots, \theta_{M}(\mathrm{k})\right)$,

$$
\begin{aligned}
& \bar{A}_{d, i}{ }^{\prime}(\mathrm{k})=\mathrm{A}_{\mathrm{d}, \mathrm{i}}-\mathrm{D}_{\theta \mathrm{u}, \mathrm{i}}{ }^{\prime} \mathrm{T}_{2, \mathrm{i}} \mathrm{F}_{2, \mathrm{i}}(\mathrm{k}), \\
& \bar{B}_{d, i}(\mathrm{k})=\mathrm{A}_{\mathrm{d}, \mathrm{i}}{ }^{\prime} \mathrm{E}_{\mathrm{i}}(\mathrm{X}(\mathrm{k}+1), \mathrm{k}) \mathrm{B}_{3 \mathrm{~d}, \mathrm{i}}-\mathrm{F}_{2, \mathrm{i}}{ }^{\prime} \mathrm{T}_{2, \mathrm{i}} \mathrm{D}_{\mathrm{u}, \mathrm{i}}(\mathrm{k})+\mathrm{C}_{1 \mathrm{~d}, \mathrm{i}}{ }^{\prime} \mathrm{D}_{13 \mathrm{~d}, \mathrm{i}}
\end{aligned}
$$

and $\theta_{c, i}(\mathrm{k})$ is the 'causal' part of $\theta_{i}(\cdot)$ at time k. This $\theta_{c, i}$ is the expected value of $\theta_{i}$ over $\bar{R}_{k}$ and given by

$$
\begin{aligned}
& \theta_{c, i}(1)=\bar{A}_{d, i}{ }^{\prime}(1) \mathrm{E}_{\mathrm{i}}\left(\theta_{c}(1+1), 1\right)+\bar{B}_{d, i}(1) \mathrm{r}_{\mathrm{d}}(1), \mathrm{k}+1 \leq \mathrm{l} \leq \mathrm{k}+\mathrm{h}, \\
& \theta_{c, i}(\mathrm{k}+\mathrm{h}+1)=0 \text { if } \mathrm{k}+\mathrm{h} \leq \mathrm{N}-1 \\
& \theta_{c, i}(\mathrm{k}+\mathrm{h}+1)=\mathrm{C}_{1 \mathrm{~d}, \mathrm{i}}{ }^{\prime} \mathrm{D}_{13 \mathrm{~d}, \mathrm{i}} \mathrm{r}_{\mathrm{d}}(\mathrm{N}), \mathrm{k}+\mathrm{h}=\mathrm{N}
\end{aligned}
$$

where $\mathrm{E}_{\mathrm{i}}\left(\theta_{c}(\mathrm{k}+1), \mathrm{k}\right)=\sum_{j=1}^{M} p_{d, i j}(k) \theta_{c, j}(\mathrm{k}+1)$ and $\theta_{c}(\mathrm{k})=\left(\theta_{c, 1}(\mathrm{k}), \cdots, \theta_{c, M}(\mathrm{k})\right)$.

Moreover, the optimal value of the performance index is

$$
\begin{aligned}
\mathrm{J}_{\mathrm{dN}}\left(\mathrm{x} 0, u_{d}^{*}, \mathrm{r}_{\mathrm{d}}\right)=\operatorname{tr}\left\{Q_{i_{0}} \mathrm{X}_{i_{0}}\right\}+\alpha_{i_{0}}(0)+\mathrm{E}\left\{E_{\overline{R_{0}}}\left\{2 \theta_{i_{0}}{ }^{\prime} \mathrm{x}_{0}\right\}\right\} \\
\quad+\mathrm{E}\left\{\sum_{k=0}^{N-1} E_{\overline{R_{k}}}\left\{\left|T_{2, m(k)}^{1 / 2} \mathrm{D}_{\theta \mathrm{u}, \mathrm{m}(\mathrm{k})}(\mathrm{k}) \mathrm{E}_{\mathrm{m}(\mathrm{k})}\left(\theta_{c}^{-}(\mathrm{k}+1), \mathrm{k}\right)\right|^{2}\right\}\right\}+\bar{J}_{d}\left(\mathrm{r}_{\mathrm{d}}\right)
\end{aligned}
$$

where $\quad \theta_{c, m(k)}^{-}(\mathrm{k})=\theta_{m(k)}(\mathrm{k})-\theta_{c, m(k)}(\mathrm{k}), \mathrm{k} \in[0, \mathrm{~N}]$,

$$
\begin{aligned}
& \mathrm{E}_{\mathrm{i}}\left(\theta_{c}^{-}(\mathrm{k}+1), \mathrm{k}\right)=\sum_{j=1}^{M} p_{d, i j}(k) \theta_{c, j}^{-}(\mathrm{k}+1), \theta_{c}^{-}(\mathrm{k})=\left(\theta_{c, 1}^{-}(\mathrm{k}), \cdots, \theta_{c, M}^{-}(\mathrm{k})\right) \text { and } \\
& \bar{J}_{d}\left(\mathrm{r}_{\mathrm{d}}\right)=\mathrm{E}\left\{E_{\overline{R_{N}}}\left\{\left|\mathrm{D}_{13 \mathrm{~d}, \mathrm{~m}(\mathrm{~N})}(\mathrm{N}) \mathrm{r}_{\mathrm{d}}(\mathrm{N})\right|^{2}\right\}\right\}+\mathrm{E}\left\{\sum _ { k = 0 } ^ { N - 1 } E _ { \overline { R _ { k } } } \left\{-\left|T_{2, m(k)}^{1 / 2} \mathrm{D}_{\theta \mathrm{u}, \mathrm{m}(\mathrm{k})}(\mathrm{k}) \mathrm{E}_{\mathrm{m}(\mathrm{k})}(\theta(\mathrm{k}+1), \mathrm{k})\right|^{2}\right.\right. \\
& -2 \mathrm{E}_{\mathrm{m}(\mathrm{k})}\left(\theta^{\prime}(\mathrm{k}+1), \mathrm{k}\right) \mathrm{D}_{\theta \mathrm{u}, \mathrm{m}(\mathrm{k})}{ }^{\prime} \mathrm{T}_{2, m(k)} \mathrm{D}_{\mathrm{u}, \mathrm{m}(\mathrm{k})}(\mathrm{k}) \mathrm{r}_{\mathrm{d}}(\mathrm{k}) \\
& \left.\left.+2 \mathrm{E}_{\mathrm{m}(\mathrm{k})}\left(\theta^{\prime}(\mathrm{k}+1), \mathrm{k}\right) \mathrm{B}_{3 \mathrm{~d}, \mathrm{~m}(\mathrm{k})}(\mathrm{k}) \mathrm{r}_{\mathrm{d}}(\mathrm{k})+\mathrm{J}_{\mathrm{d}, \mathrm{k}, \mathrm{m}(\mathrm{k})}\left(\mathrm{r}_{\mathrm{d}}\right)\right\}\right\} \text {, }
\end{aligned}
$$




$$
\begin{aligned}
\mathrm{J}_{\mathrm{d}, \mathrm{k}, \mathrm{m}(\mathrm{k})}\left(\mathrm{r}_{\mathrm{d}}\right)=\mathrm{r}_{\mathrm{d}}{ }^{\prime}(\mathrm{k}) & {\left[-\mathrm{D}_{\mathrm{u}, \mathrm{m}(\mathrm{k})}{ }^{\prime} \mathrm{T}_{2, m(k)} \mathrm{D}_{\mathrm{u}, \mathrm{m}(\mathrm{k})}(\mathrm{k})+\mathrm{B}_{3 \mathrm{~d}, \mathrm{~m}(\mathrm{k})}{ }^{\prime} \mathrm{E}_{\mathrm{m}(\mathrm{k})}(\mathrm{X}(\mathrm{k}+1), \mathrm{k}) \mathrm{B}_{3 \mathrm{~d}, \mathrm{~m}(\mathrm{k})}\right.} \\
+ & \left.+\mathrm{D}_{13 \mathrm{~d}, \mathrm{~m}(\mathrm{k})}{ }^{\prime} \mathrm{D}_{13 \mathrm{~d}, \mathrm{~m}(\mathrm{k})}\right] \mathrm{r}_{\mathrm{d}}(\mathrm{k}) .
\end{aligned}
$$

(Proof) See the appendix 1 .

Remark 3.2 Note that each dynamics (6) of $\theta_{c, i}$, which composes the compensator introducing the preview information, is coupled with the others. It corresponds to the characteristic that the Riccati difference equations (3) are coupled with each other, which give the necessary and sufficient conditions for the solvability of the stochastic optimal tracking problem by state feedback.

Next we consider the following two extreme cases according to the information structures (preview lengths) of $r_{d}$ :

i. Stochastic Optimal Tracking of Causal $\left\{\mathrm{r}_{\mathrm{d}}(\cdot)\right\}$ :

In this case, $\left\{r_{d}(k)\right\}$ is measured on-line, i.e., at time $k, r_{d}(l)$ is known only for $l \leq k$.

ii. Stochastic Optimal Tracking of Noncausal $\left\{\mathrm{r}_{\mathrm{d}}(\cdot)\right\}$ :

In this case, the signal $\left\{\mathrm{r}_{\mathrm{d}}(\mathrm{k})\right\}$ is assumed to be known a priori for the whole time interval $\mathrm{k} \in[0, \mathrm{~N}]$.

Utilizing the optimal control strategy for the stochastic optimal tracking problem in Theorem 3.1, we present the solutions to these two extreme cases.

Corollary 3.1 Consider the system (1) and the performance index (2). Suppose A1, A2 and A3. Then each of the stochastic optimal tracking problems for (1) and (2) is solvable by state feedback if and only if there exist matrices $\mathrm{X}_{\mathrm{i}}(\mathrm{k}) \geq \mathrm{O}$ and scalar functions $\alpha_{i}(\mathrm{k}), \mathrm{i}=1, \cdots, \mathrm{M}$, satisfying the conditions $\mathrm{X}_{\mathrm{i}}(\mathrm{N})=\mathrm{C}_{1 \mathrm{~d}, \mathrm{i}^{\prime}}(\mathrm{N}) \mathrm{C}_{1 \mathrm{~d}, \mathrm{i}}(\mathrm{N})$ and $\alpha_{i}(\mathrm{~N})=0$ such that the coupled Riccati difference equations (3) and the coupled scalar equations (4) hold over $[0, N]$. Moreover, the following results hold using the three types of gains

$$
K_{d, x, i}(k)=F_{2, i}(k), K_{r d, i}(k)=D_{u, i}(k) \text { and } K_{d, \theta, i}(k)=D_{\theta u, i}(k) \text { for } i=1, \cdots, M \text {. }
$$

i. The control law for the Stochastic Optimal Tracking of Causal $\left\{\mathrm{r}_{\mathrm{d}}(\cdot)\right\}$ is

$$
\mathrm{u}_{\mathrm{d}, \mathrm{s} 1}(\mathrm{k})=\mathrm{K}_{\mathrm{d}, \mathrm{x}, \mathrm{i}}(\mathrm{k}) \mathrm{x}(\mathrm{k})+\mathrm{K}_{\mathrm{rd}, \mathrm{i}}(\mathrm{k}) \mathrm{r}_{\mathrm{d}}(\mathrm{k}) \text { for } \mathrm{i}=1, \ldots, \mathrm{M}
$$

and the value of the performance index is

$$
\begin{aligned}
& \mathrm{J}_{\mathrm{dN}}\left(\mathrm{x}_{0}, \mathrm{u}_{\mathrm{d}, \mathrm{s} 1}, \mathrm{r}_{\mathrm{d}}\right)=\operatorname{tr}\left\{Q_{i_{0}} \mathrm{X}_{i_{0}}\right\}+\alpha_{i_{0}}(0)+\mathrm{E}\left\{E_{\overline{R_{0}}}\left\{2 \theta_{i_{0}}{ }^{\prime} \mathrm{x}_{0}\right\}\right\} \\
& +\mathrm{E}\left\{\sum_{k=0}^{N-1} E_{\overline{R_{k}}}\left\{\left|T_{2, m(k)}^{1 / 2} \mathrm{D}_{\theta \mathrm{u}, \mathrm{m}(\mathrm{k})}(\mathrm{k}) \mathrm{E}_{\mathrm{m}(\mathrm{k})}(\theta(\mathrm{k}+1), \mathrm{k})\right|^{2}\right\}\right\}+\bar{J}_{d}\left(\mathrm{r}_{\mathrm{d}}\right) .
\end{aligned}
$$

ii. The control law for the Stochastic Optimal Tracking of Noncausal $\left\{r_{d}(\cdot)\right\}$ is

$$
\mathrm{u}_{\mathrm{d}, \mathrm{s} 2}(\mathrm{k})=\mathrm{K}_{\mathrm{d}, \mathrm{x}, \mathrm{i}}(\mathrm{k}) \mathrm{x}(\mathrm{k})+\mathrm{K}_{\mathrm{rd}, \mathrm{i}}(\mathrm{k}) \mathrm{r}_{\mathrm{d}}(\mathrm{k})+\mathrm{K}_{\mathrm{d}, \theta, \mathrm{i}}(\mathrm{k}) \mathrm{E}_{\mathrm{i}}(\theta(\mathrm{k}+1), \mathrm{k}) \text { for } \mathrm{i}=1, \cdots, \mathrm{M}
$$

with $\theta_{i}(\cdot)$ given by (5) and the value of the performance index is

$$
\mathrm{J}_{\mathrm{dN}}\left(\mathrm{x}_{0}, \mathrm{u}_{\mathrm{d}, \mathrm{s} 2,}, \mathrm{r}_{\mathrm{d}}\right)=\operatorname{tr}\left\{Q_{i_{0}} \mathrm{X}_{i_{0}}\right\}+\alpha_{i_{0}}(0)+2 \theta_{i_{0}}{ }^{\prime} \mu_{0}+\bar{J}_{d}\left(\mathrm{r}_{\mathrm{d}}\right) .
$$

(Proof)

i. In this causal case, the control law is not affected by the effects of any preview information and so $\theta_{c}(\mathrm{k})=0$ for all $\mathrm{k} \in[0, \mathrm{~N}]$ since the each dynamics of $\theta_{c, i}$ becomes 
autonomous. As a result we obtain $\theta(\mathrm{k})=\theta_{c}^{-}(\mathrm{k})$ for all $\mathrm{k} \in[0, \mathrm{~N}]$. Therefore we obtain the value of the performance index $\mathrm{J}_{\mathrm{dN}}\left(\mathrm{x}_{0}, \mathrm{u}_{\mathrm{d}, \mathrm{s} 1}, \mathrm{r}_{\mathrm{d}}\right)$.

ii. In this noncausal case, $\mathrm{h}=\mathrm{N}-\mathrm{k}$ and (5) and (6) becomes identical. As a result we obtain $\theta(\mathrm{k})=\theta_{c}(\mathrm{k})$ for all $\mathrm{k} \in[0, \mathrm{~N}]$. Therefore we obtain $\theta_{c}^{-}(\mathrm{k})=0$ for all $\mathrm{k} \in[0, \mathrm{~N}]$ and the value of the performance index $\mathrm{J}_{\mathrm{dN}}\left(\mathrm{x}_{0}, \mathrm{u}_{\mathrm{d}, \mathrm{s} 2}, \mathrm{r}_{\mathrm{d}}\right)$. Notice that, in this case, we can obtain the deterministic value of $\theta_{i_{0}}(0)$ using the information of $\left\{\mathrm{r}_{\mathrm{d}}(\cdot)\right\}$ until the final time $\mathrm{N}$ and so the term $\mathrm{E}\left\{E_{\overline{R_{0}}}\left\{2 \theta_{i_{0}}{ }^{\prime} x_{0}\right\}\right\}$ in the right hand side of (7) reduces to $2 \theta_{i_{0}}{ }^{\prime} \mu_{0}$. (Q.E.D.)

\section{Output feedback case}

In this section, we consider the output feedback problems.

We first assume the following conditions:

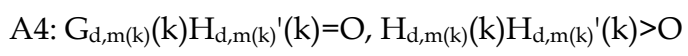

By the transformation

$$
\bar{u}_{d, c}(\mathrm{k}):=\mathrm{u}_{\mathrm{d}}(\mathrm{k})-\mathrm{D}_{\mathrm{u}, \mathrm{i}}(\mathrm{k}) \mathrm{r}_{\mathrm{d}}(\mathrm{k})-\mathrm{D}_{\theta \mathrm{u}, \mathrm{i}}(\mathrm{k}) \mathrm{E}_{\mathrm{i}}\left(\theta_{c}(\mathrm{k}+1), \mathrm{k}\right)
$$

and the coupled difference equations (3) and (4), we can rewrite the performance index as follows:

$$
\begin{aligned}
\mathrm{J}_{\mathrm{dN}}\left(\mathrm{x}_{0}, \bar{u}_{d, c}, \mathrm{r}_{\mathrm{d}}\right)= & \operatorname{tr}\left\{Q_{i_{0}} \mathrm{X}_{i_{0}}\right\}+\alpha_{i_{0}}(0) \\
& +\mathrm{E}\left\{E_{\overline{R_{0}}}\left\{2^{\prime} \theta_{i_{0}} \mathrm{x}_{0}\right\}\right\} \\
& +\mathrm{E}\left\{\sum_{k=0}^{N-1} E_{\overline{R_{k}}}\left\{\left|\bar{u}_{d, c}(\mathrm{k})-\mathrm{F}_{2, \mathrm{~m}(\mathrm{k})}(\mathrm{k}) \times(\mathrm{k})-\mathrm{D}_{\theta \mathrm{u}, \mathrm{m}(\mathrm{k})}(\mathrm{k}) \mathrm{E}_{\mathrm{m}(\mathrm{k})}\left(\theta_{c}^{-}(\mathrm{k}+1), \mathrm{k}\right)\right|_{T_{2, m(k)}}^{2}\right\}\right\} \\
& +\bar{J}_{d}\left(\mathrm{r}_{\mathrm{d}}\right)
\end{aligned}
$$

and the dynamics can be written as follows:

where

$$
\mathrm{x}(\mathrm{k}+1)=\mathrm{A}_{\mathrm{d}, \mathrm{m}(\mathrm{k})}(\mathrm{k}) \mathrm{x}(\mathrm{k})+\mathrm{G}_{\mathrm{d}, \mathrm{m}(\mathrm{k})}(\mathrm{k}) \omega_{\mathrm{d}}(\mathrm{k})+\mathrm{B}_{2 \mathrm{~d}, \mathrm{~m}(\mathrm{k})}(\mathrm{k}) \bar{u}_{d, c}(\mathrm{k})+\bar{r}_{d, c}(\mathrm{k})
$$

$$
\bar{r}_{d, c}(\mathrm{k})=\mathrm{B}_{2 \mathrm{~d}, \mathrm{~m}(\mathrm{k})}\left\{\mathrm{D}_{\mathrm{u}, \mathrm{m}(\mathrm{k})}(\mathrm{k}) \mathrm{r}_{\mathrm{d}}(\mathrm{k})+\mathrm{D}_{\theta \mathrm{u}, \mathrm{m}(\mathrm{k})}(\mathrm{k}) \mathrm{E}_{\mathrm{m}(\mathrm{k})}\left(\theta_{c}(\mathrm{k}+1), \mathrm{k}\right)\right\}+\mathrm{B}_{3 \mathrm{~d}, \mathrm{~m}(\mathrm{k})}(\mathrm{k}) \mathrm{r}_{\mathrm{d}}(\mathrm{k}) .
$$

For this plant dynamics, consider the controller

$$
\begin{aligned}
\hat{x}_{e}(\mathrm{k}+1)= & \mathrm{A}_{\mathrm{d}, \mathrm{m}(\mathrm{k})}(\mathrm{k}) \hat{x}_{e}(\mathrm{k})+\mathrm{B}_{2 \mathrm{~d}, \mathrm{~m}(\mathrm{k})}(\mathrm{k}) \bar{u}_{d, c^{*}}(\mathrm{k}) \\
& +\mathrm{B}_{3 \mathrm{~d}, \mathrm{~m}(\mathrm{k})}(\mathrm{k}) \bar{r}_{d, c}(\mathrm{k})-\mathrm{M}_{\mathrm{m}(\mathrm{k})}(\mathrm{k})\left[\mathrm{y}(\mathrm{k})-\mathrm{C}_{2 \mathrm{~d}, \mathrm{~m}(\mathrm{k})} \hat{x}_{e}(\mathrm{k})\right] \\
& \hat{x}_{e}(0)=\mathrm{E}\left\{E_{\overline{R_{0}}}\left\{\mathrm{x}_{0}\right\}\right\}=\mu_{0}, \bar{u}_{d, c^{*}}(\mathrm{k})=\mathrm{F}_{2, \mathrm{~m}(\mathrm{k})}(\mathrm{k}) \hat{x}_{e}(\mathrm{k})
\end{aligned}
$$

where $\mathrm{M}_{\mathrm{m}(\mathrm{k})}$ are the controller gains to decide later, using the solutions of another coupled Riccati equations introduced below.

Define the error variable

$$
\mathrm{e}(\mathrm{k}):=\mathrm{x}(\mathrm{k})-\hat{x}_{e}(\mathrm{k})
$$


and the error dynamics is as follows:

$$
\begin{aligned}
\mathrm{e}(\mathrm{k}+1) & =\mathrm{A}_{\mathrm{d}, \mathrm{m}(\mathrm{k})}(\mathrm{k}) \mathrm{e}(\mathrm{k})+\mathrm{G}_{\mathrm{d}, \mathrm{m}(\mathrm{k})}(\mathrm{k}) \omega_{\mathrm{d}}(\mathrm{k})+\mathrm{M}_{\mathrm{m}(\mathrm{k})}(\mathrm{k})\left[\mathrm{y}(\mathrm{k})-\mathrm{C}_{2 \mathrm{~d}, \mathrm{~m}(\mathrm{k})} \hat{x}_{e}(\mathrm{k})\right] \\
& =\left[\mathrm{A}_{\mathrm{d}, \mathrm{m}(\mathrm{k})}+\mathrm{M}_{\mathrm{m}(\mathrm{k})} \mathrm{C}_{2 \mathrm{~d}, \mathrm{~m}(\mathrm{k})}\right](\mathrm{k}) \mathrm{e}(\mathrm{k})+\left[\mathrm{G}_{\mathrm{d}, \mathrm{m}(\mathrm{k})}+\mathrm{M}_{\mathrm{m}(\mathrm{k})} \mathrm{H}_{\mathrm{d}, \mathrm{m}(\mathrm{k})}\right](\mathrm{k}) \omega_{\mathrm{d}}(\mathrm{k})
\end{aligned}
$$

Note that this error dynamics does not depend on the exogenous inputs $u_{d}$ nor $r_{d}$. Our objective is to design the controller gain $\mathrm{M}_{\mathrm{m}(\mathrm{k})}$ which minimizes

$$
\begin{aligned}
& \mathrm{J}_{\mathrm{dN}}\left(\mathrm{x}_{0}, \bar{u}_{d, c^{*}}, \mathrm{r}_{\mathrm{d}}\right) \operatorname{tr}\left\{Q_{i_{0}} \mathrm{X}_{i_{0}}\right\}+\alpha_{i_{0}}(0)+\mathrm{E}\left\{E_{\overline{R_{0}}}\left\{2 \theta_{i_{0}}{ }^{\prime} \mathrm{x}_{0}\right\}\right\} \\
&+\mathrm{E}\left\{\sum _ { k = 0 } ^ { N - 1 } E _ { \overline { R _ { k } } } \left\{\mid \mathrm{F}_{2, \mathrm{~m}(\mathrm{k})}(\mathrm{k}) \mathrm{e}(\mathrm{k})\right.\right. \\
&\left.\left.\quad-\left.\mathrm{D}_{\theta \mathrm{u}, \mathrm{m}(\mathrm{k})}(\mathrm{k}) \mathrm{E}_{\mathrm{m}(\mathrm{k})}\left(\theta_{c}^{-}(\mathrm{k}+1), \mathrm{k}\right)\right|_{T_{2, m(k)}} ^{2}\right\}\right\}+\bar{J}_{d}\left(\mathrm{r}_{\mathrm{d}}\right)
\end{aligned}
$$

Notice that $\mathrm{e}(\mathrm{k})$ and $\mathrm{E}_{\mathrm{m}(\mathrm{k})}\left(\theta_{c}^{-}(\mathrm{k}+1), \mathrm{k}\right)$ are mutually independent.

We decide the gain matrices $M_{i}(k), i=1, \ldots, M$ by designing the LMMSE filter such that $\sum_{k=0}^{N-1} \mathrm{E}\left\{E_{\overline{R_{k}}}\left\{|\mathrm{e}(\mathrm{k})|^{2}\right\}\right\}$ is minimized. Now we consider the following coupled Riccati difference equations and the initial conditions.

$$
\begin{array}{r}
\mathrm{Y}_{\mathrm{j}}(\mathrm{k}+1)=\sum_{i \in J(k)} p_{d, i j}\left[\mathrm{~A}_{\mathrm{d}, \mathrm{i}}{ }^{\prime} \mathrm{Y}_{\mathrm{i}}(\mathrm{k}) \mathrm{A}_{\mathrm{d}, \mathrm{i}}-\mathrm{A}_{\mathrm{d}, \mathrm{i}} \mathrm{Y}_{\mathrm{i}}(\mathrm{k}) \mathrm{C}_{2 \mathrm{~d}, \mathrm{i}}{ }^{\prime}\left(\mathrm{H}_{\mathrm{d}, \mathrm{i}} \mathrm{H}_{\mathrm{d}, \mathrm{i}}{ }^{\prime} \Pi_{\mathrm{i}}(\mathrm{k})\right.\right. \\
\left.\left.+\mathrm{C}_{2 \mathrm{~d}, \mathrm{i}} \mathrm{Y}_{\mathrm{i}}(\mathrm{k}) \mathrm{C}_{2 \mathrm{~d}, \mathrm{i}}{ }^{\prime}\right)^{-1} \mathrm{C}_{2 \mathrm{~d}, \mathrm{i}} \mathrm{Y}_{\mathrm{i}}(\mathrm{k}) \mathrm{A}_{\mathrm{d}, \mathrm{i}}{ }^{\prime}+\Pi_{\mathrm{i}}(\mathrm{k}) \mathrm{G}_{\mathrm{d}, \mathrm{i}} \mathrm{G}_{\mathrm{d}, \mathrm{i}}{ }^{\prime}\right], \\
\mathrm{Y}_{\mathrm{i}}(0)=\Pi_{\mathrm{i}}(0)\left(Q_{i_{0}}-\mu_{0} \mu_{0}{ }^{\prime}\right)
\end{array}
$$

where

$\Pi_{\mathrm{i}}(\mathrm{k}):=\mathrm{P}\{\mathrm{m}(\mathrm{k})=\mathrm{i}\}, \sum_{j=1}^{M} p_{d, i j}(k) \Pi_{\mathrm{i}}=\Pi_{\mathrm{j}}, \sum_{i=1}^{M} \Pi_{\mathrm{i}}(\mathrm{k})=1, \mathrm{~J}(\mathrm{k}):=\left\{\mathrm{i} \in \mathrm{N} ; \Pi_{\mathrm{i}}(\mathrm{k})>0\right\}$.

These equations are also called the filtering coupled Riccati difference equations [Costa \& Tuesta (2003)].

Now since

$$
\left.\mathrm{E}\left\{E_{\overline{R_{0}}}\{\mathrm{e}(0)\}\right\}=\mathrm{E}\left\{E_{\overline{R_{0}}}\left\{\mathrm{x}_{0}\right\}-\mathrm{E}\left\{E_{\overline{R_{0}}}\left\{\mathrm{x}_{0}\right\}\right\}\right\}=\mathrm{E}\left\{E_{\overline{R_{0}}} \mathrm{x}_{0}\right\}\right\}-\mu_{0}=0
$$

and $\bar{r}_{d, c}(0)$ is deterministic if $\mathrm{r}_{\mathrm{d}}(\mathrm{l})$ is known at all $\mathrm{l} \in[0, \mathrm{k}+\mathrm{h}]$,

$$
\mathrm{E}\left\{E_{\overline{R_{0}}}\left\{\mathrm{e}(0) \bar{r}_{d, c^{\prime}}(0) 1_{\{\mathrm{m}(0)=\mathrm{i}\}}\right\}\right\}=\Pi_{\mathrm{i}}(0) \mathrm{E}\left\{E_{\overline{R_{0}}}\{\mathrm{e}(0)\}\right\} \bar{r}_{d, c^{\prime}(0)=\mathrm{O}}
$$

and so we obtain, for each $\mathrm{k} \in[0, \mathrm{~N}]$,

$$
\mathrm{E}\left\{E_{\overline{R_{k}}}\left\{\mathrm{e}(\mathrm{k}) \bar{r}_{d, c^{\prime}}(\mathrm{k}) 1_{\{\mathrm{m}(\mathrm{k})=\mathrm{i}\}}\right\}\right\}=\Pi_{\mathrm{i}}(\mathrm{k}) \mathrm{E}\left\{E_{\overline{R_{k}}}\{\mathrm{e}(\mathrm{k})\}\right\} \bar{r}_{d, c^{\prime}}(\mathrm{k})=\mathrm{O} .
$$

Namely there exist no couplings between $\mathrm{e}(\cdot)$ and $\bar{r}_{d, c}(\cdot)$. The development of $\mathrm{e}(\cdot)$ on time $\mathrm{k}$ is independent of the development of $\bar{r}_{d, c}(\cdot)$ on time $\mathrm{k}$. Then we can show the following orthogonal property as [Theorem 5.3 in (Costa et al. (2005)) or Theorem 2 in (Costa \& Tuesta (2003))] by induction on $\mathrm{k}$ (See the appendix 2). 


$$
\mathrm{E}\left\{E_{\overline{R_{k}}}\left\{\mathrm{e}(\mathrm{k}) \hat{x}_{e}{ }^{\prime}(\mathrm{k}) 1_{\{\mathrm{m}(\mathrm{k})=\mathrm{i}\}}\right\}\right\}=\mathrm{O}
$$

Moreover define

$$
\left.\bar{Y}_{i}(\mathrm{k}):=\mathrm{E}\left\{E_{\overline{R_{k}}} \mathrm{e}(\mathrm{k}) \mathrm{e}^{\prime}(\mathrm{k}) 1_{\{\mathrm{m}(\mathrm{k})=\mathrm{i}\}}\right\}\right\}
$$

and then we can show

$$
\mathrm{Y}_{\mathrm{i}}(\mathrm{k})=\bar{Y}_{i}(\mathrm{k})
$$

From all these results (orthogonal properties), as the case of $r_{d}(\cdot) \equiv 0$, using the solutions of the coupled difference Riccati equations, it can be shown that the gains $M_{m(k)}$ minimizing $J_{d N}$ are decided as follows (cf. [Costa \& Tuesta (2003); Costa et al. (2005)]):

$$
M_{i}(k)=\left\{\begin{array}{l}
-A_{d, i} Y_{i}(k) C_{2 d, i}{ }^{\prime}\left(H_{d, i} H_{d, i}{ }^{\prime} \Pi_{i}(k)+C_{2 d, i} Y_{i}(k) C_{2 d, i}{ }^{\prime}\right)^{-1} \text { for } \mathrm{i} \in J(k) \\
0 \text { for } i \in J(k)
\end{array}\right.
$$

Finally the following theorem, which gives the solution of the output feedback problem, holds.

Theorem 4.1 Consider the system (1) and the performance index (2). Suppose A1, A2, A3 and A4. Then an optimal control strategy which, gives the solution of the Stochastic Optimal Fixed-Preview Tracking Problem by Output Feedback for (1) and (2) is given by the dynamic controller (8) with the gains (11) using the solutions of the two types of the coupled Riccati difference equations (3) with $X_{i}(N)=C_{1 d, i}{ }^{\prime}(N) C_{1 d, i}(N)$ and (9) with $Y_{i}(0)=\Pi_{i}(0)\left(Q_{i_{0}}\right.$ $\left.\mu_{0} \mu_{0}{ }^{\prime}\right)$.

Remark 4.1 Notice that

$$
\begin{aligned}
\mathrm{E}\left\{E_{\overline{R_{k}}}\left\{\left|\mathrm{z}_{\mathrm{d}}(\mathrm{k})\right|^{2}\right\}\right\}= & \sum_{i=1}^{M} \operatorname{tr}\left\{\mathrm{C}_{1 \mathrm{~d}, \mathrm{i}} \mathrm{C}_{1 \mathrm{~d}, \mathrm{i}}{ }^{\prime} \mathrm{E}\left\{E_{\overline{R_{k}}}\left\{\mathrm{x}(\mathrm{k}) \mathrm{x}^{\prime}(\mathrm{k}) 1_{\{\mathrm{m}(\mathrm{k})=\mathrm{i}\}}\right\}\right\}\right\} \\
& +\mathrm{E}\left\{E_{\overline{R_{k}}}\left\{\left|\mathrm{D}_{12 \mathrm{~d}, \mathrm{~m}(\mathrm{k})}(\mathrm{k}) \mathrm{u}_{\mathrm{d}}(\mathrm{k})\right|^{2}+2 \mathrm{x}^{\prime}(\mathrm{k}) \mathrm{C}_{1 \mathrm{~d},{ }^{\prime}} \mathrm{D}_{13 \mathrm{~d}, \mathrm{i}_{\mathrm{d}}}(\mathrm{k})\right\}\right\} .
\end{aligned}
$$

Then, with regard to the performance index, the following result holds.

$$
\begin{gathered}
\mathrm{E}\left\{E_{\overline{R_{k}}}\left\{\left|\mathrm{z}_{\mathrm{d}}(\mathrm{k})\right|^{2}\right\}\right\}=\mathrm{E}\left\{E_{\overline{R_{k}}}\left\{\left|\hat{z}_{e}(\mathrm{k})\right|^{2}\right\}\right\}+\sum_{i=1}^{M} \operatorname{tr}\left\{\mathrm{C}_{1 \mathrm{~d}, \mathrm{i}} \mathrm{Y}_{\mathrm{i}}(\mathrm{k}) \mathrm{C}_{1 \mathrm{~d}, \mathrm{i}}{ }^{\prime}\right\} \\
+\mathrm{E}\left\{\sum_{i=1}^{M} E_{\bar{R}_{k}}\left\{2 \mathrm{e}^{\prime}(\mathrm{k}) \mathrm{C}_{1 \mathrm{~d},{ }^{\prime}}{ }^{\prime} \mathrm{D}_{13 \mathrm{~d}, \mathrm{i}} \mathrm{r}_{\mathrm{d}}(\mathrm{k}) 1_{\{\mathrm{m}(\mathrm{k})=\mathrm{i}\}}\right\}\right\}
\end{gathered}
$$

where

$$
\hat{z}_{e}(\mathrm{k})=\mathrm{C}_{1 \mathrm{~d}, \mathrm{~m}(\mathrm{k})} \hat{x}_{e}(\mathrm{k})+\mathrm{D}_{12 \mathrm{~d}, \mathrm{~m}(\mathrm{k})}(\mathrm{k}) \mathrm{u}_{\mathrm{d}}(\mathrm{k})+\mathrm{D}_{13 \mathrm{~d}, \mathrm{~m}(\mathrm{k})}(\mathrm{k}) \mathrm{r}_{\mathrm{d}}(\mathrm{k})
$$

and we have used the property

$$
\begin{gathered}
\mathrm{E}\left\{E_{\overline{R_{k}}}\left\{\mathrm{x}(\mathrm{k}) \mathrm{x}^{\prime}(\mathrm{k}) 1_{\{\mathrm{m}(\mathrm{k})=\mathrm{i}\}}\right\}\right\}=\mathrm{E}\left\{E_{\overline{R_{k}}}\left\{\mathrm{e}(\mathrm{k}) \mathrm{e}^{\prime}(\mathrm{k}) 1_{\{\mathrm{m}(\mathrm{k})=\mathrm{i}\}}\right\}\right\}+\mathrm{E}\left\{E_{\overline{R_{k}}}\left\{\hat{x}_{e}(\mathrm{k}) \hat{x}_{e}{ }^{\prime}(\mathrm{k}) 1_{\{\mathrm{m}(\mathrm{k})=\mathrm{i}\}}\right\}\right\} \\
=\mathrm{Y}_{\mathrm{i}}(\mathrm{k})+\mathrm{E}\left\{E_{\overline{R_{k}}}\left\{\hat{x}_{e}(\mathrm{k}) \hat{x}_{e}{ }^{\prime}(\mathrm{k}) 1_{\{\mathrm{m}(\mathrm{k})=\mathrm{i}\}}\right\}\right\}
\end{gathered}
$$

by the orthogonal property (10). 
Note that the second and third terms in the right hand side do not depend on the input $u_{d}$.

Then we obtain

$$
\begin{aligned}
\mathrm{J}_{\mathrm{dN}}\left(\mathrm{x}_{0}, \mathrm{u}_{\mathrm{d},} \mathrm{r}_{\mathrm{d}}\right)= & \mathrm{E}\left\{\sum _ { k = 0 } ^ { N } \left[E_{\overline{R_{k}}}\left\{\left|\hat{z}_{e}(\mathrm{k})\right|^{2}\right\}+\sum_{i=1}^{M} \operatorname{tr}\left\{\mathrm{C}_{1 \mathrm{~d}, \mathrm{i}} \mathrm{Y}_{\mathrm{i}}(\mathrm{k}) \mathrm{C}_{1 \mathrm{~d}, \mathrm{i}}{ }^{\prime}\right\}\right.\right. \\
& \left.\left.+\sum_{i=1}^{M} E_{\bar{R}_{k}}\left\{2 \mathrm{e}^{\prime}(\mathrm{k}) \mathrm{C}_{1 \mathrm{~d}, \mathrm{i}}{ }^{\prime} \mathrm{D}_{13 \mathrm{~d}, \mathrm{i}} \mathrm{r}_{\mathrm{d}}(\mathrm{k}) 1_{\{\mathrm{m}(\mathrm{k})=\mathrm{i}\}}\right\}\right\}\right] \\
& +\mid \mathrm{C}_{1 \mathrm{~d}, \mathrm{~m}(\mathrm{~N})} \mathrm{x}(\mathrm{N})+\mathrm{D}_{\left.13 \mathrm{~d},\left.\mathrm{~m}(\mathrm{~N}) \mathrm{r}_{\mathrm{d}}(\mathrm{N})\right|^{2}\right\}}
\end{aligned}
$$

Therefore minimizing (12) is equivalent to minimizing $\mathrm{E}\left\{\sum_{k=0}^{N} E_{\overline{R_{k}}}\left\{\left|\hat{z}_{e}(\mathrm{k})\right|^{2}\right\}\right.$ subject to the dynamics

$$
\hat{x}_{e}(\mathrm{k}+1)=\mathrm{A}_{\mathrm{d}, \mathrm{m}(\mathrm{k})}(\mathrm{k}) \hat{x}_{e}(\mathrm{k})+\mathrm{B}_{2 \mathrm{~d}, \mathrm{~m}(\mathrm{k})}(\mathrm{k}) \bar{u}_{d, c^{*}}(\mathrm{k})+\bar{r}_{d, c}(\mathrm{k})-\mathrm{M}_{\mathrm{m}(\mathrm{k})}(\mathrm{k}) \mathrm{v}(\mathrm{k}), \hat{x}_{e}(0)=E_{\overline{R_{0}}}\left\{\mathrm{x}_{0}\right\}=\mu_{0}
$$

where

$$
\mathrm{v}(\mathrm{k})=\mathrm{y}(\mathrm{k})-\mathrm{C}_{2 \mathrm{~d}, \mathrm{~m}(\mathrm{k})} \hat{x}_{e}(\mathrm{k})
$$

and $\bar{u}_{d, c^{*}}(\mathrm{k})$ is the state feedback controller with the form $\mathrm{K}_{\mathrm{d}, \mathrm{x}, \mathrm{i}}(\mathrm{k}) \hat{x}_{e}(\mathrm{k})+\mathrm{K}_{\mathrm{rd}, \mathrm{i}}(\mathrm{k}) \mathrm{r}_{\mathrm{d}}(\mathrm{k})+\mathrm{K}_{\mathrm{d}, \theta, \mathrm{i}}(\mathrm{k}) \mathrm{E}_{\mathrm{i}}(\theta(\mathrm{k}+1), \mathrm{k})$ for some gains $\mathrm{K}_{\mathrm{d}, \mathrm{x}, \mathrm{i}}, \mathrm{K}_{\mathrm{rd}, \mathrm{i}}$ and $\mathrm{K}_{\mathrm{d}, \theta, \mathrm{i} .}$ Note that the term $\mathrm{M}_{\mathrm{m}(\mathrm{k})}(\mathrm{k}) \mathrm{v}(\mathrm{k})$ plays the same role as the "noise" term $\mathrm{G}_{\mathrm{d}, \mathrm{m}(\mathrm{k})}(\mathrm{k}) \omega_{\mathrm{d}}(\mathrm{k})$ of the plant dynamics in the state feedback case.

Remark 4.2 As the case of $r_{d}(\cdot) \equiv 0$, the separation principle holds in the case of $r_{d}(\cdot) \equiv 0$. Namely we can design the state feedback gains $F_{2, m(k)}(k)$ and the filter gains $M_{m(k)}$ separately.

Utilizing the optimal control strategy for the stochastic optimal tracking problem in Theorem 4.1, we present the solutions to the two extreme cases.

Corollary 4.1 Consider the system (1) and the performance index (2). Suppose A1, A2, A3 and A4. Then optimal control strategies by output feedback for the two extreme cases are as follows using the solutions of the two types of the coupled Riccati difference equations (3) with $X_{i}(N)=C_{1 d, i}(N) C_{1 d, i}(N)$ and (9) with $Y_{i}(0)=\Pi_{i}(0)\left(Q_{i_{0}}-\mu_{0} \mu_{0}{ }^{\prime}\right)$ :

i. The control law by output feedback for the Stochastic Optimal Tracking of Causal $\left\{\mathrm{r}_{\mathrm{d}}(\cdot)\right\}$ is

$$
\begin{aligned}
& \hat{x}_{e}(\mathrm{k}+1)= \mathrm{A}_{\mathrm{d}, \mathrm{m}(\mathrm{k})}(\mathrm{k}) \hat{x}_{e}(\mathrm{k})+\mathrm{B}_{2 \mathrm{~d}, \mathrm{~m}(\mathrm{k})}(\mathrm{k}) \bar{u}_{d, 1^{*}}(\mathrm{k})+\bar{r}_{d, 1}(\mathrm{k})-\mathrm{M}_{\mathrm{m}(\mathrm{k})}(\mathrm{k}) \mathrm{v}(\mathrm{k}) \\
& \hat{x}_{e}(0)=\mu_{0} \\
& \bar{u}_{d, 1}(\mathrm{k}):=\mathrm{u}_{\mathrm{d}}(\mathrm{k})-\mathrm{D}_{\mathrm{u}, \mathrm{i}}(\mathrm{k}) \mathrm{r}_{\mathrm{d}}(\mathrm{k}) \bar{u}_{d, 1^{*}}(\mathrm{k})=\mathrm{F}_{2, \mathrm{~m}(\mathrm{k})}(\mathrm{k}) \hat{x}_{e}(\mathrm{k}) \\
& \bar{r}_{d, 1}(\mathrm{k})=\mathrm{B}_{2 \mathrm{~d}, \mathrm{~m}(\mathrm{k})} \mathrm{D}_{\mathrm{u}, \mathrm{m}(\mathrm{k})}(\mathrm{k}) \mathrm{r}_{\mathrm{d}}(\mathrm{k})+\mathrm{B}_{3 \mathrm{~d}, \mathrm{~m}(\mathrm{k})}(\mathrm{k}) \mathrm{r}_{\mathrm{d}}(\mathrm{k})
\end{aligned}
$$

and the value of the performance index is

$$
\begin{aligned}
\mathrm{J}_{\mathrm{dN}}\left(\mathrm{x}_{0}, \bar{u}_{d, 1^{*},} \mathrm{r}_{\mathrm{d}}\right)= & \operatorname{tr}\left\{Q_{i_{0}} \mathrm{X}_{i_{0}}\right\}+\alpha_{i_{0}}(0)+\mathrm{E}\left\{E_{\overline{R_{0}}}\left\{2 \theta_{i_{0}}{ }^{\prime} \mathrm{x}_{0}\right\}\right\} \\
& +\mathrm{E}\left\{\sum _ { k = 0 } ^ { N - 1 } E _ { \overline { R _ { k } } } \left\{\mid \mathrm{F}_{2, \mathrm{~m}(\mathrm{k})}(\mathrm{k}) \mathrm{e}(\mathrm{k})\right.\right. \\
& \left.\left.-\left.\mathrm{D}_{\theta \mathrm{u}, \mathrm{m}(\mathrm{k})}(\mathrm{k}) \mathrm{E}_{\mathrm{m}(\mathrm{k})}(\theta(\mathrm{k}+1), \mathrm{k})\right|_{T_{2, \mathrm{~m}(k)}} ^{2}\right\}\right\}+\bar{J}_{d}\left(\mathrm{r}_{\mathrm{d}}\right)
\end{aligned}
$$


ii. The control law by output feedback for the Stochastic Optimal Tracking of Noncausal $\left\{r_{d}(\cdot)\right\}$ is

$$
\begin{aligned}
\hat{x}_{e}(\mathrm{k}+1)= & \mathrm{A}_{\mathrm{d}, \mathrm{m}(\mathrm{k})}(\mathrm{k}) \hat{x}_{e}(\mathrm{k})+\mathrm{B}_{2 \mathrm{~d}, \mathrm{~m}(\mathrm{k})}(\mathrm{k}) \bar{u}_{d, 2^{*}}(\mathrm{k})+\bar{r}_{d, 2}(\mathrm{k})-\mathrm{M}_{\mathrm{m}(\mathrm{k})}(\mathrm{k}) \mathrm{v}(\mathrm{k}) \\
& \hat{x}_{e}(0)=\mu_{0} \\
\bar{u}_{d, 2}(\mathrm{k}):= & \mathrm{u}_{\mathrm{d}}(\mathrm{k})-\mathrm{D}_{\mathrm{u}, i}(\mathrm{k}) \mathrm{r}_{\mathrm{d}}(\mathrm{k})-\mathrm{D}_{\theta \mathrm{u}, \mathrm{i}}(\mathrm{k}) \mathrm{E}_{\mathrm{i}}(\theta(\mathrm{k}+1), \mathrm{k}) \\
\bar{u}_{d, 2^{*}}(\mathrm{k})= & \mathrm{F}_{2, \mathrm{~m}(\mathrm{k})}(\mathrm{k}) \hat{x}_{e}(\mathrm{k}) \\
\bar{r}_{d, 2}(\mathrm{k})= & \mathrm{B}_{2 \mathrm{~d}, \mathrm{~m}(\mathrm{k})}\left\{\mathrm{D}_{\mathrm{u}, \mathrm{m}(\mathrm{k})}(\mathrm{k}) \mathrm{r}_{\mathrm{d}}(\mathrm{k})+\mathrm{D}_{\theta \mathrm{u}, \mathrm{m}(\mathrm{k})}(\mathrm{k}) \mathrm{E}_{\mathrm{m}(\mathrm{k})}(\theta(\mathrm{k}+1), \mathrm{k})\right\}+\mathrm{B}_{3 \mathrm{~d}, \mathrm{~m}(\mathrm{k})}(\mathrm{k}) \mathrm{r}_{\mathrm{d}}(\mathrm{k})
\end{aligned}
$$

and the value of the performance index is

$$
\mathrm{J}_{\mathrm{dN}}\left(\mathrm{x}_{0}, \bar{u}_{d, 2^{*}}, \mathrm{r}_{\mathrm{d}}\right)=\operatorname{tr}\left\{Q_{i_{0}} \mathrm{X}_{i_{0}}\right\}+\alpha_{i_{0}}(0)+2 \theta_{i_{0}}{ }^{\prime} \mu_{0}+\mathrm{E}\left\{\sum_{k=0}^{N-1} E_{\overline{R_{k}}}\left\{\left|\mathrm{~F}_{2, \mathrm{~m}(\mathrm{k})}(\mathrm{k}) \mathrm{e}(\mathrm{k})\right|_{T_{2, m(k)}}^{2}\right\}\right\}+\bar{J}_{d}\left(\mathrm{r}_{\mathrm{d}}\right) .
$$

(Proof) As the state feedback cases, $\theta_{c}(\mathrm{k})=0$, i.e., $\theta(\mathrm{k})=\theta_{c}^{-}(\mathrm{k})$ for all $\mathrm{k} \in[0, \mathrm{~N}]$ in the case $\mathrm{i}$ ), and $\theta(\mathrm{k})=\theta_{c}(\mathrm{k})$, i.e., $\theta_{c}^{-}(\mathrm{k})=0$ for all $\mathrm{k} \in[0, \mathrm{~N}]$ in the case ii).

\section{Numerical examples}

In this section, we study numerical examples to demonstrate the effectiveness of the presented stochastic LQ preview tracking design theory.

We consider the following two mode systems and assume that the system parameters are as follows. (cf. [Cohen \& Shaked (1997); Shaked \& Souza (1995)].):

$$
\begin{aligned}
& x(k+1)=A_{d, m(k)}(k) x(k)+G_{d}(k) \omega_{d}(k)+B_{2 d}(k) u_{d}(k)+B_{3 d, m(k)}(k) r_{d}(k) \\
& x(0)=x_{0}, m(0)=i_{0,} m(k)=1,2 \\
& z_{d}(k)=C_{1 d, m(k)}(k) x(k)+D_{12 d, m(k)}(k) u_{d}(k)+D_{13 d, m(k)}(k) r_{d}(k) \\
& y(k)=C_{2 d, m(k)}(k) x(k)+H_{d, m(k)}(k) \omega_{d}(k)
\end{aligned}
$$

where

Mode 1:

Mode 2:

$$
\begin{aligned}
& \mathrm{A}_{\mathrm{d}, 1}=\left[\begin{array}{cc}
0 & 1 \\
-0.8 & 1.6
\end{array}\right], \mathrm{A}_{\mathrm{d}, 2}=\left[\begin{array}{cc}
0 & 1 \\
1.6 & 1.6
\end{array}\right], \mathrm{G}_{\mathrm{d}}=\left[\begin{array}{c}
0 \\
0.1
\end{array}\right], \mathrm{B}_{2 \mathrm{~d}}=\left[\begin{array}{l}
0 \\
1
\end{array}\right], \\
& \mathrm{B}_{3 \mathrm{~d}, 1}=\left[\begin{array}{c}
1.5 \\
0
\end{array}\right], \mathrm{B}_{3 \mathrm{~d}, 2}=\left[\begin{array}{c}
1.8 \\
0
\end{array}\right], \\
& \mathrm{C}_{1 \mathrm{~d}, 1}=\left[\begin{array}{cc}
-0.5 & 0.2 \\
0 & 0
\end{array}\right], \mathrm{C}_{1 \mathrm{~d}, 2}=\left[\begin{array}{cc}
-0.5 & 0.1 \\
0 & 0
\end{array}\right], \mathrm{D}_{12 \mathrm{~d}}=\left[\begin{array}{c}
0 \\
0.1
\end{array}\right], \mathrm{D}_{13 \mathrm{~d}}=\left[\begin{array}{c}
-1.0 \\
0
\end{array}\right]
\end{aligned}
$$

Let

$$
\mathrm{P}_{\mathrm{d}}=\left[\begin{array}{ll}
0.3 & 0.7 \\
0.6 & 0.4
\end{array}\right]
$$

be a stationary transition matrix of $\{\mathrm{m}(\mathrm{k})\}$. We set $\mathrm{x}_{0}=\operatorname{col}(0,0)$ and $\mathrm{i}_{0}=1$. 
Then we introduce the following objective function.

$$
\begin{aligned}
\mathrm{J}_{\mathrm{dN}}\left(\mathrm{x}_{0}, \mathrm{u}_{\mathrm{d}}, \mathrm{r}_{\mathrm{d}}\right):=\mathrm{E}\left\{\sum_{k=0}^{N} E_{\overline{R_{k}}}\left\{\left|\mathrm{C}_{1 \mathrm{~d}, \mathrm{~m}(\mathrm{k})}(\mathrm{k}) \mathrm{x}(\mathrm{k})+\mathrm{D}_{13 \mathrm{~d}, \mathrm{~m}(\mathrm{k})}(\mathrm{k}) \mathrm{r}_{\mathrm{d}}(\mathrm{k})\right|^{2}\right\}\right\} \\
+0.01 \mathrm{E}\left\{\sum_{k=0}^{N-1} E_{\overline{R_{k}}}\left\{\left|\mathrm{u}_{\mathrm{d}}(\mathrm{k})\right|^{2}\right\}\right\}
\end{aligned}
$$

By the term $B_{3 d, i}(k) r_{d}(k), i=1,2$, the tracking performance can be expected to be improved as [Cohen \& Shaked (1997); Shaked \& Souza (1995)] and so on. The paths of $m(k)$ are generated randomly, and the performances are compared under the same condition, that is, the same set of the paths so that the performances can be easily compared.

We consider the whole system (13) with mode transition rate $P_{d}$ over the time interval $k \in[0,100]$. For this system (13) with the rate matrix $P_{d}$, we apply the results of the optimal tracking design theory by output feedback for $r_{d}(k)=0.5 \sin (n k / 20)$ and $r_{d}(k)=0.5 \sin (n k / 100)$ with various step lengths of preview, and show the simulation results for sample paths.

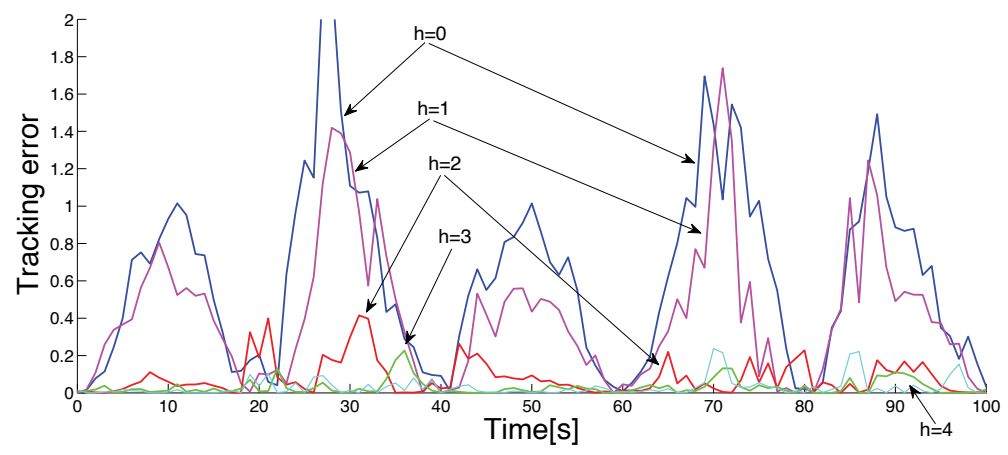

Fig. $1(a) \cdot r_{d}(k)=0.5 \sin (n k / 20)$

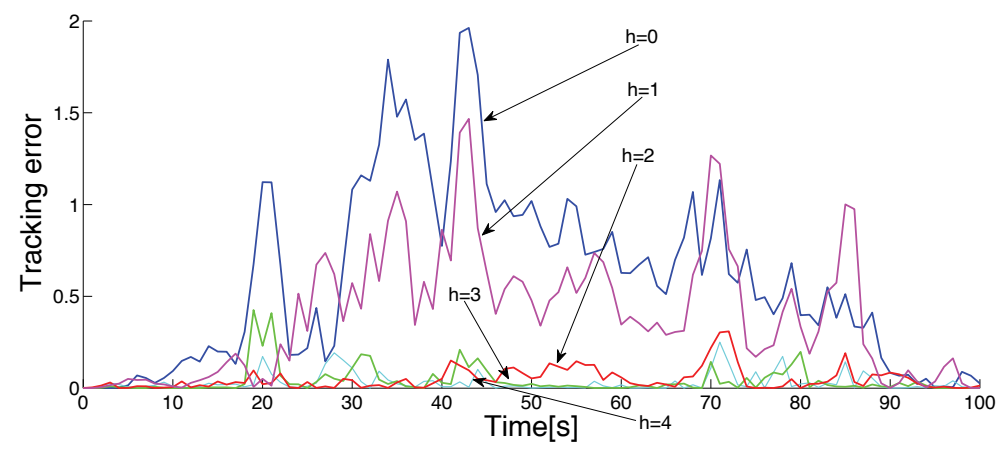

Fig. $1(b) \cdot r_{d}(k)=0.5 \sin (n k / 100)$

Fig. 1 . The whole system consisting of mode 1 and mode 2 : The errors of tracking for various preview lengths 
It is shown in Fig. 1(a) for $r_{d}(k)=0.5 \sin (n k / 20)$ and Fig. $1(b) r_{d}(k)=0.5 \sin (n k / 100)$ that increasing the preview steps from $h=0$ to $h=1,2,3,4$ improves the tracking performance. In fact, the square values $\left|C_{1 d, i}(k) x(k)+D_{13 d}(k) r_{d}(k)\right|^{2}$ of the tracking errors are shown in Fig. 1(a) and (b) and it is clear the tracking error decreases as increasing the preview steps by these figures.

\section{Conclusion}

In this paper we have studied the stochastic linear quadratic (LQ) optimal tracking control theory considering the preview information by state feedback and output feedback for the linear discrete-time Markovian jump systems affected by the white noises, which are a class of stochastic switching systems, and verified the effectiveness of the design theory by numerical examples. In order to solve the output feedback problems, we have introduced the LMMSE filters adapted to the effects of preview feedforward compensation. In order to design the output feedback controllers, we need the solutions of two types of coupled Riccati difference equations, i.e., the ones to decide the state feedback gains and the ones to decide the filter gains. These solutions of two types of coupled Riccati difference equations can be obtained independently i.e., the separation principle holds. Correspondingly the compensators introducing the preview information of the reference signal are coupled with each other. This is the very important research result in this paper.

We have considered both of the cases of full and partial observation. However, in these cases, we have considered the situations that the switching modes are observable over whole time interval. The construction of the design theory for the case that the switching modes are unknown is a very important further research issue.

\section{Appendix 1. Proof of Proposition 3.1}

(Proof of Proposition 3.1)

\section{Sufficiency:}

Let $\mathrm{X}_{\mathrm{i}}(\mathrm{k})>\mathrm{O}$ and $\alpha_{i}, \mathrm{i}=1, \ldots, \mathrm{M}$, be solutions to (3) and (4) over $[0, \mathrm{~N}]$ such that $\mathrm{X}_{\mathrm{i}}(\mathrm{N})=\mathrm{C}_{1 \mathrm{~d}, \mathrm{i}}{ }^{\prime}(\mathrm{N}) \mathrm{C}_{1 \mathrm{~d}, \mathrm{i}}(\mathrm{N})$ and $\alpha_{i}(\mathrm{~N})=0$.

Define

$$
\begin{aligned}
\phi_{k, m(k)}:=E_{\overline{R_{k+1}}}\left\{\mathrm{E}\left\{\mathrm{x}^{\prime}(\mathrm{k}+1) \mathrm{X}_{\mathrm{m}(\mathrm{k}+1)}(\mathrm{k}+1) \mathrm{x}(\mathrm{k}+1)+\alpha_{\mathrm{m}(\mathrm{k}+1)}(\mathrm{k}+1) \mid \mathrm{x}(\mathrm{k}), \mathrm{m}(\mathrm{k})\right\}\right\} \\
-E_{\overline{R_{k}}}\left\{\mathrm{x}^{\prime}(\mathrm{k}) \mathrm{X}_{\mathrm{m}(\mathrm{k})} \mathrm{x}(\mathrm{k})+\alpha_{\mathrm{m}(\mathrm{k})}(\mathrm{k})\right\}
\end{aligned}
$$

We first consider the case of $r_{d}(\cdot) \equiv 0$. Then the following equalities hold by the assumptions A3.

$$
\begin{aligned}
\mathrm{E}\left\{\mathrm{x}^{\prime}(\mathrm{k}+1) \mathrm{X}_{\mathrm{m}(\mathrm{k}+1)}(\mathrm{k}+1) \mathrm{x}(\mathrm{k}+1)+\alpha \mathrm{m}(\mathrm{k}+1)\right. & (\mathrm{k}+1) \mid \mathrm{x}(\mathrm{k}), \mathrm{m}(\mathrm{k})\} \\
=\mathrm{E}\{ & \left(\mathrm{A}_{\mathrm{d}, \mathrm{m}(\mathrm{k})}(\mathrm{k}) \mathrm{x}(\mathrm{k})+\mathrm{G}_{\mathrm{d}, \mathrm{m}(\mathrm{k})}(\mathrm{k}) \omega_{\mathrm{d}}(\mathrm{k})+\mathrm{B}_{2 \mathrm{~d}, \mathrm{~m}(\mathrm{k})}(\mathrm{k}) \mathrm{u}_{\mathrm{d}}(\mathrm{k})\right)^{\prime} \\
& \times \mathrm{X}_{\mathrm{m}(\mathrm{k}+1)}(\mathrm{k}+1)\left(\mathrm{A}_{\mathrm{d}, \mathrm{m}(\mathrm{k})}(\mathrm{k}) \mathrm{x}(\mathrm{k})+\mathrm{G}_{\mathrm{d}, \mathrm{m}(\mathrm{k})}(\mathrm{k}) \omega_{\mathrm{d}}(\mathrm{k})+\mathrm{B}_{2 \mathrm{~d}, \mathrm{~m}(\mathrm{k})}(\mathrm{k}) \mathrm{u}_{\mathrm{d}}(\mathrm{k})\right) \\
& \left.+\alpha_{\mathrm{m}(\mathrm{k}+1)}(\mathrm{k}+1) \mid \mathrm{x}(\mathrm{k}), \mathrm{m}(\mathrm{k})\right\}
\end{aligned}
$$




$$
\begin{aligned}
=\left(\mathrm{A}_{\mathrm{d}, \mathrm{m}(\mathrm{k})}(\mathrm{k}) \mathrm{x}(\mathrm{k})+\mathrm{B}_{2 \mathrm{~d}, \mathrm{~m}(\mathrm{k})}(\mathrm{k}) \mathrm{u}_{\mathrm{d}}(\mathrm{k})\right)^{\prime} \\
\quad \times \mathrm{E}_{\mathrm{m}(\mathrm{k})}(\mathrm{X}(\mathrm{k}+1), \mathrm{k})\left(\mathrm{A}_{\mathrm{d}, \mathrm{m}(\mathrm{k})}(\mathrm{k}) \mathrm{x}(\mathrm{k})+\mathrm{B}_{2 \mathrm{~d}, \mathrm{~m}(\mathrm{k})}(\mathrm{k}) \mathrm{u}_{\mathrm{d}}(\mathrm{k})\right) \\
\quad+\sum_{j=1}^{M} \operatorname{tr}\left\{\mathrm{G}_{\mathrm{d}, \mathrm{i}}(\mathrm{k}) \mathrm{X}_{i}(\mathrm{k}) \mathrm{G}_{\mathrm{d}, \mathrm{i}}{ }^{\prime}(\mathrm{k}) \mathrm{E}_{\mathrm{i}}(\mathrm{X}(\mathrm{k}+1), \mathrm{k})\right\}+\mathrm{E}\left\{\alpha_{\mathrm{m}(\mathrm{k}+1)}(\mathrm{k}+1) \mid \mathrm{x}(\mathrm{k}), \mathrm{m}(\mathrm{k})\right\}
\end{aligned}
$$

It can be shown that the following equality holds, using the system (1) and the coupled Riccati equations (3) and the coupled scalar equations (4). ([Costa et al. (2005); Fragoso (1989)])

$$
\phi_{k, m(k)}=E_{\overline{R_{k}}}\left\{-\left|\mathrm{z}_{\mathrm{d}}(\mathrm{k})\right|^{2}+\left|T_{2, m(k)}^{1 / 2}(\mathrm{k})\left[\mathrm{u}_{\mathrm{d}}(\mathrm{k})-\mathrm{F}_{2, \mathrm{~m}(\mathrm{k})}(\mathrm{k}) \mathrm{x}(\mathrm{k})\right]\right|^{2}\right\}
$$

Moreover, in the genaral case that $r_{d}(\cdot)$ is arbitrary, we have the following equality.

$$
\begin{gathered}
\phi_{k, m(k)}=E_{\overline{R_{k}}}\left\{-\left|\mathrm{z}_{\mathrm{d}}(\mathrm{k})\right|^{2}+\left|T_{2, m(k)}^{1 / 2}(\mathrm{k})\left[\mathrm{u}_{\mathrm{d}}(\mathrm{k})-\mathrm{F}_{2, \mathrm{~m}(\mathrm{k})}(\mathrm{k}) \mathrm{x}(\mathrm{k})\right]-\mathrm{D}_{\mathrm{u}, \mathrm{m}(\mathrm{k})}(\mathrm{k}) \mathrm{r}_{\mathrm{d}}(\mathrm{k})\right|^{2}\right. \\
\left.+2 \mathrm{x}^{\prime}(\mathrm{k}) \bar{B}_{d, m(k)}(\mathrm{k}) \mathrm{r}_{\mathrm{d}}(\mathrm{k})+\mathrm{J}_{\mathrm{d}, \mathrm{k}, \mathrm{m}(\mathrm{k})}\left(\mathrm{r}_{\mathrm{d}}\right)\right\}
\end{gathered}
$$

Notice that, in the right hand side of this equality, $\mathrm{J}_{\mathrm{d}, \mathrm{k}, \mathrm{m}(\mathrm{k})}\left(\mathrm{r}_{\mathrm{d}}\right)$, which means the tracking error without considering the effect of the preview information, is added.

Now introducing the vector $\theta_{m(k)}$, which can include some preview information of the tracking signals,

$$
\begin{aligned}
E_{\overline{R_{k+1}}}\left\{\mathrm{E}\left\{\theta_{m(k+1)}{ }^{\prime}(\mathrm{k}+1) \mathrm{x}(\mathrm{k}+1) \mid \mathrm{x}(\mathrm{k}), \mathrm{m}(\mathrm{k})\right\}\right\}-E_{\overline{R_{k}}}\left\{\theta_{m(k)}{ }^{\prime}(\mathrm{k}) \mathrm{x}(\mathrm{k})\right\} \\
=E_{\overline{R_{k}}}\left\{\mathrm { E } _ { \mathrm { m } ( \mathrm { k } ) } ( \theta ^ { \prime } ( \mathrm { k } + 1 ) , \mathrm { k } ) \left(\mathrm{~A}_{\mathrm{d}, \mathrm{m}(\mathrm{k})}(\mathrm{k}) \mathrm{x}(\mathrm{k})+\mathrm{G}_{\mathrm{d}, \mathrm{m}(\mathrm{k})}(\mathrm{k}) \omega_{\mathrm{d}}(\mathrm{k})\right.\right. \\
\left.\left.+\mathrm{B}_{2 \mathrm{~d}, \mathrm{~m}(\mathrm{k})}(\mathrm{k}) \mathrm{u}_{\mathrm{d}}(\mathrm{k})+\mathrm{B}_{3 \mathrm{~d}, \mathrm{~m}(\mathrm{k})}(\mathrm{k}) \mathrm{r}_{\mathrm{d}}(\mathrm{k})\right)\right\}-E_{\overline{R_{k+1}}}\left\{\theta_{m(k)}{ }^{\prime}(\mathrm{k}) \mathrm{x}(\mathrm{k})\right\}
\end{aligned}
$$

Then we obtain

$$
\begin{aligned}
& \phi_{k, m(k)}+ 2\left\{E_{\overline{R_{k+1}}}\left\{\mathrm{E}\left\{\theta_{m(k+1)}{ }^{\prime}(\mathrm{k}+1) \mathrm{x}(\mathrm{k}+1) \mid \mathrm{x}(\mathrm{k}), \mathrm{m}(\mathrm{k})\right\}\right\}-E_{\overline{R_{k}}}\left\{\theta_{m(k)}{ }^{\prime}(\mathrm{k}) \mathrm{x}(\mathrm{k})\right\}\right\} \\
&=E_{\overline{R_{k}}}\left\{-\left|\mathrm{z}_{\mathrm{d}}(\mathrm{k})\right|^{2}+\left|T_{2, m(k)}^{1 / 2}(\mathrm{k})\left[\mathrm{u}_{\mathrm{d}}(\mathrm{k})-\mathrm{F}_{2, \mathrm{~m}(\mathrm{k})}(\mathrm{k}) \mathrm{x}(\mathrm{k})-\mathrm{D}_{\mathrm{u}, \mathrm{m}(\mathrm{k})}(\mathrm{k}) \mathrm{r}_{\mathrm{d}}(\mathrm{k})\right]\right|^{2}\right. \\
&\left.+2 \mathrm{x}^{\prime}(\mathrm{k}) \bar{B}_{d, m(k)}(\mathrm{k}) \mathrm{r}_{\mathrm{d}}(\mathrm{k})+\mathrm{J}_{\mathrm{d}, \mathrm{k}, \mathrm{m}(\mathrm{k})}\left(\mathrm{r}_{\mathrm{d}}\right)\right\} \\
&+ 2 E_{\overline{R_{k}}}\left\{\left\{\mathrm { E } _ { \mathrm { m } ( \mathrm { k } ) } ( \theta ^ { \prime } ( \mathrm { k } + 1 ) , \mathrm { k } ) \left(\mathrm{~A}_{\mathrm{d}, \mathrm{m}(\mathrm{k})}(\mathrm{k}) \mathrm{x}(\mathrm{k})+\mathrm{G}_{\mathrm{d}, \mathrm{m}(\mathrm{k})}(\mathrm{k}) \omega_{\mathrm{d}}(\mathrm{k})\right.\right.\right. \\
&\left.\left.\left.+\mathrm{B}_{2 \mathrm{~d}, \mathrm{~m}(\mathrm{k})}(\mathrm{k}) \mathrm{u}_{\mathrm{d}}(\mathrm{k})+\mathrm{B}_{3 \mathrm{~d}, \mathrm{~m}(\mathrm{k})}(\mathrm{k}) \mathrm{r}_{\mathrm{d}}(\mathrm{k})\right)\right\}-E_{\overline{R_{k+1}}}\left\{\theta_{m(k)}(\mathrm{k}) \mathrm{x}(\mathrm{k})\right\}\right\} \\
&=E_{\overline{R_{k}}}\left\{-\left|\mathrm{z}_{\mathrm{d}}(\mathrm{k})\right|^{2}+\mid T_{2, m(k)}^{1 / 2}(\mathrm{k})\left[\mathrm{u}_{\mathrm{d}}(\mathrm{k})-\mathrm{F}_{2, \mathrm{~m}(\mathrm{k})}(\mathrm{k}) \mathrm{x}(\mathrm{k})-\mathrm{D}_{\mathrm{u}, \mathrm{m}(\mathrm{k})}(\mathrm{k}) \mathrm{r}_{\mathrm{d}}(\mathrm{k})\right.\right. \\
&\left.\left.-\mathrm{D}_{\theta \mathrm{u}, \mathrm{m}(\mathrm{k})}(\mathrm{k}) \mathrm{E}_{\mathrm{m}(\mathrm{k})}(\theta(\mathrm{k}+1), \mathrm{k})\right]\left.\right|^{2}+\bar{J}_{d, k, m(k)}\left(\mathrm{r}_{\mathrm{d}}\right)\right\}
\end{aligned}
$$

where

$$
\theta_{i}(\mathrm{k})=\bar{A}_{d, i}{ }^{\prime}(\mathrm{k}) \mathrm{E}_{\mathrm{i}}(\theta(\mathrm{k}+1), \mathrm{k})+\bar{B}_{d, i}(\mathrm{k}) \mathrm{r}_{\mathrm{d}}(\mathrm{k})
$$


to get rid of the mixed terms of $\mathrm{r}_{\mathrm{d}}$ and $\mathrm{x}$, or $\theta_{m(k)}$ and $\mathrm{x} . \bar{J}_{d, k, m(k)}\left(\mathrm{r}_{\mathrm{d}}\right)$ means the tracking error including the preview information vector $\theta$ and can be expressed by

$$
\begin{gathered}
\left.\bar{J}_{d, k, m(k)}\left(\mathrm{r}_{\mathrm{d}}\right)=-\mid T_{2, m(k)}^{1 / 2} \mathrm{D}_{\theta \mathrm{u}, \mathrm{m}(\mathrm{k})}(\mathrm{k}) \mathrm{E}_{\mathrm{m}(\mathrm{k})}(\theta(\mathrm{k}+1), \mathrm{k})\right]\left.\right|^{2} \\
-\mathrm{E}_{\mathrm{m}(\mathrm{k})}\left(\theta^{\prime}(\mathrm{k}+1), \mathrm{k}\right) \mathrm{D}_{\theta \mathrm{u}, \mathrm{m}(\mathrm{k})}{ }^{\prime} \mathrm{T}_{2, \mathrm{~m}(\mathrm{k})} \mathrm{D}_{\mathrm{u}, \mathrm{m}(\mathrm{k})}(\mathrm{k}) \mathrm{r}_{\mathrm{d}}(\mathrm{k}) \\
+2 \mathrm{E}_{\mathrm{m}(\mathrm{k})}\left(\theta^{\prime}(\mathrm{k}+1), \mathrm{k}\right) \mathrm{B}_{3 \mathrm{~d}, \mathrm{~m}(\mathrm{k})} \mathrm{r}_{\mathrm{d}}(\mathrm{k})+\mathrm{J}_{\mathrm{d}, \mathrm{k}, \mathrm{m}(\mathrm{k})}\left(\mathrm{r}_{\mathrm{d}}\right)
\end{gathered}
$$

Taking the sum of the quantities (14) from $k=0$ to $k=N-1$ and adding $E\left\{\mid C_{1 d, m(N)}(N) x(N)+\right.$ $\left.\left.\mathrm{D}_{13 \mathrm{~d}, \mathrm{~m}(\mathrm{~N})}(\mathrm{N}) \mathrm{r}_{\mathrm{d}}(\mathrm{N})\right|^{2}\right\}$ and taking the expectation $\mathrm{E}\{\}$,

$$
\begin{gathered}
\sum_{k=0}^{N-1} \mathrm{E}\left\{E_{\overline{R_{k}}}\left\{\left|\mathrm{z}_{\mathrm{d}}(\mathrm{k})\right|^{2}\right\}\right\}+\mathrm{E}\left\{\left|\mathrm{C}_{1 \mathrm{~d}, \mathrm{~m}(\mathrm{~N})}(\mathrm{N}) \mathrm{x}(\mathrm{N})+\mathrm{D}_{13 \mathrm{~d}, \mathrm{~m}(\mathrm{~N})}(\mathrm{N}) \mathrm{r}_{\mathrm{d}}(\mathrm{N})\right|^{2}\right\} \\
+\sum_{k=0}^{N-1} \mathrm{E}\left\{\phi_{k, m(k)}+2\left\{E_{\overline{R_{k+1}}}\left\{\mathrm{E}\left\{\theta_{m(k+1)}{ }^{\prime}(\mathrm{k}+1) \mathrm{x}(\mathrm{k}+1) \mid \mathrm{x}(\mathrm{k}), \mathrm{m}(\mathrm{k})\right\}\right\}\right.\right. \\
\left.\left.\quad-E_{\overline{R_{k}}}\left\{\theta_{m(k)}{ }^{\prime}(\mathrm{k}) \mathrm{x}(\mathrm{k})\right\}\right\} \mid \mathrm{x}(\mathrm{k}), \mathrm{m}(\mathrm{k})\right\} \\
=\sum_{k=0}^{N-1} \mathrm{E}\left\{E_{\overline{R_{k}}}\left\{\left|\hat{u}_{d}(\mathrm{k})-\mathrm{D}_{\theta \mathrm{u}, \mathrm{m}(\mathrm{k})}(\mathrm{k}) \mathrm{E}_{\mathrm{m}(\mathrm{k})}(\theta(\mathrm{k}+1), \mathrm{k})\right|_{T_{2, m(k)}(k)}^{2}\right\}\right. \\
+\mathrm{E}\left\{\left|\mathrm{C}_{1 \mathrm{~d}, \mathrm{~m}(\mathrm{~N})}(\mathrm{N}) \mathrm{x}(\mathrm{N})+\mathrm{D}_{13 \mathrm{~d}, \mathrm{~m}(\mathrm{~N})}(\mathrm{N}) \mathrm{r}_{\mathrm{d}}(\mathrm{N})\right|^{2}\right\} \\
+\sum_{k=0}^{N-1} \mathrm{E}\left\{E_{\overline{R_{k}}}\left\{\bar{J}_{d, k, m(k)}\left(\mathrm{r}_{\mathrm{d}}\right)\right\}\right\}
\end{gathered}
$$

where

$$
\hat{u}_{d}(\mathrm{k})=\mathrm{u}_{\mathrm{d}}(\mathrm{k})-\mathrm{F}_{2, \mathrm{~m}(\mathrm{k})}(\mathrm{k}) \times(\mathrm{k})-\mathrm{D}_{\mathrm{u}, \mathrm{m}(\mathrm{k})}(\mathrm{k}) \mathrm{r}_{\mathrm{d}}(\mathrm{k})
$$

Since the left hand side reduces to

$$
\begin{aligned}
\sum_{k=0}^{N-1} \mathrm{E}\left\{E_{\overline{R_{k}}}\left\{\left|\mathrm{z}_{\mathrm{d}}(\mathrm{k})\right|^{2}\right\}\right\}+\mathrm{E}\left\{\left|\mathrm{C}_{1 \mathrm{~d}, \mathrm{~m}(\mathrm{~N})}(\mathrm{N}) \mathrm{x}(\mathrm{N})+\mathrm{D}_{13 \mathrm{~d}, \mathrm{~m}(\mathrm{~N})}(\mathrm{N}) \mathrm{r}_{\mathrm{d}}(\mathrm{N})\right|^{2}\right\} \\
+\mathrm{E}\left\{2 \theta_{m(N)}{ }^{\prime}(\mathrm{N}) \mathrm{x}(\mathrm{N})+\mathrm{x}^{\prime}(\mathrm{N}) \mathrm{X}_{\mathrm{m}(\mathrm{N})}(\mathrm{N}) \mathrm{x}(\mathrm{N})+\alpha_{m(N)}(\mathrm{N})\right\} \\
+\mathrm{E}\left\{E_{\overline{R_{0}}}\left\{-2 \theta_{i_{0}}{ }^{\prime}(0) \mathrm{x}(0)-\mathrm{x}^{\prime}(0) X_{i_{0}}(0) \mathrm{x}(0)-\alpha_{i_{0}}(0)\right\}\right\}
\end{aligned}
$$

noticing that the equality

$$
\begin{gathered}
E_{\overline{R_{N}}}\left\{\mathrm{E}\left\{\mathrm{x}^{\prime}(\mathrm{N}) \mathrm{X}_{\mathrm{m}(\mathrm{N})}(\mathrm{N}) \mathrm{x}(\mathrm{N})+\alpha_{m(N)}(\mathrm{N})+2 \theta_{m(N)}{ }^{\prime}(\mathrm{N}) \mathrm{x}(\mathrm{N}) \mid \mathrm{x}(\mathrm{l}), \mathrm{m}(\mathrm{l})\right\}\right\} \\
-E_{\overline{R_{l}}}\left\{\mathrm{x}^{\prime}(\mathrm{l}) \mathrm{X}_{\mathrm{m}(\mathrm{l})} \mathrm{x}(\mathrm{l})+\alpha_{m(l)}(\mathrm{l})+2 \theta_{m(l)}{ }^{\prime}(\mathrm{l}) \mathrm{x}(\mathrm{l})\right\} \\
=\sum_{k=l}^{N-1} \mathrm{E}\left\{E _ { \overline { R _ { k + 1 } } } \left\{\mathrm { E } \left\{\mathrm{x}^{\prime}(\mathrm{k}+1) \mathrm{X}_{\mathrm{m}(\mathrm{k}+1)}(\mathrm{k}+1) \mathrm{x}(\mathrm{k}+1)+\alpha_{m(k+1)}(\mathrm{k}+1)\right.\right.\right. \\
\left.\left.+2 \theta_{m(k+1)}{ }^{\prime}(\mathrm{k}+1) \mathrm{x}(\mathrm{k}+1) \mid \mathrm{x}(\mathrm{k}), \mathrm{m}(\mathrm{k})\right\}\right\}
\end{gathered}
$$




$$
\begin{gathered}
\left.-E_{\overline{R_{k}}}\left\{\mathrm{x}^{\prime}(\mathrm{k}) \mathrm{X}_{\mathrm{m}(\mathrm{k})} \mathrm{x}(\mathrm{k})+\alpha_{m(k)}(\mathrm{k})+2 \theta_{m(k)}{ }^{\prime}(\mathrm{k}) \mathrm{x}(\mathrm{k})\right\} \mid \mathrm{x}(\mathrm{l}), \mathrm{m}(\mathrm{l})\right\} \\
=\sum_{k=1}^{N-1} \mathrm{E}\left\{\phi_{k, m(k)}\right. \\
+2\left\{E_{\overline{R_{k+1}}}\left\{\mathrm{E}\left\{\theta_{m(k+1)}{ }^{\prime}(\mathrm{k}+1) \mathrm{x}(\mathrm{k}+1) \mid \mathrm{x}(\mathrm{k}), \mathrm{m}(\mathrm{k})\right\}\right\}\right. \\
\left.\left.-\left\{E_{\overline{R_{k}}} \theta_{m(k)}{ }^{\prime}(\mathrm{k}) \mathrm{x}(\mathrm{k})\right\}\right\} \times(\mathrm{l}), \mathrm{m}(\mathrm{l})\right\}
\end{gathered}
$$

holds for $1,0 \leq 1 \leq \mathrm{N}-1$, we obtain

$$
\begin{gathered}
\mathrm{J}_{\mathrm{dN}}\left(\mathrm{x}_{0,}, \mathrm{u}_{\mathrm{d},} \mathrm{r}_{\mathrm{d}}\right)=\operatorname{tr}\left\{Q_{i_{0}} X_{i_{0}}\right\}+\alpha_{i_{0}}(0)+\mathrm{E}\left\{E_{\overline{R_{0}}}\left\{2 \theta_{i_{0}}{ }^{\prime}(0) \mathrm{x}_{0}\right\}\right\} \\
+\mathrm{E}\left\{\sum_{k=0}^{N-1} E_{\overline{R_{k}}}\left\{\left|\hat{u}_{d}(\mathrm{k})-\mathrm{D}_{\theta \mathrm{u}, \mathrm{m}(\mathrm{k})}(\mathrm{k}) \mathrm{E}_{\mathrm{m}(\mathrm{k})}(\theta(\mathrm{k}+1), \mathrm{k})\right|_{T_{2, m(k)}}^{2}(k)\right\}\right\}+\mathrm{E}\left\{\bar{J}_{d}\left(\mathrm{r}_{\mathrm{d}}\right)\right\}
\end{gathered}
$$

where we have used the terminal conditions $X_{i}(N)=C_{1 d, i}(N) C_{1 d, i}(N), \quad \theta_{i}(N)=C_{1 d, i}{ }^{\prime} D_{13 d, j} r_{d}(N)$ and $\alpha_{i}(\mathrm{~N})=0$. Note that $\bar{J}_{d}\left(\mathrm{r}_{\mathrm{d}}\right)$ is independent of $\mathrm{u}_{\mathrm{d}}$ and $\mathrm{x}_{0}$. Since the average of $\theta_{c, m(k)}^{-}(\mathrm{k})$ over $\bar{R}_{k}$ is zero, including the 'causal' part $\theta_{c, m(k)}(\mathrm{k})$ of $\theta(\cdot)$ at time k, we adopt

$$
\hat{u}_{d}^{*}(\mathrm{k})=\mathrm{D}_{\theta \mathrm{u}, \mathrm{m}(\mathrm{k})}(\mathrm{k}) \mathrm{E}_{\mathrm{m}(\mathrm{k})}\left(\theta_{c}(\mathrm{k}+1), \mathrm{k}\right)
$$

as the minimizing control strategy.

Then finally we obtain

$$
\begin{gathered}
\mathrm{J}_{\mathrm{dN}}\left(\mathrm{x}_{0}, \mathrm{u}_{\mathrm{d}}, \mathrm{r}_{\mathrm{d}}\right)=\operatorname{tr}\left\{Q_{i_{0}} X_{i_{0}}\right\}+\alpha_{i_{0}}(0)+\mathrm{E}\left\{E_{\overline{R_{0}}}\left\{2 \theta_{i_{0}}{ }^{\prime}(0) \mathrm{x}_{0}\right\}\right\} \\
+\mathrm{E}\left\{\sum_{k=0}^{N-1} E_{\overline{R_{k}}}\left\{\left|\hat{u}_{d}(\mathrm{k})-\mathrm{D}_{\theta \mathrm{u}, \mathrm{m}(\mathrm{k})}(\mathrm{k}) \mathrm{E}_{\mathrm{m}(\mathrm{k})}(\theta(\mathrm{k}+1), \mathrm{k})\right|_{T_{2, m(k)}}^{2}(k)\right\}\right\}+\mathrm{E}\left\{\bar{J}_{d}\left(\mathrm{r}_{\mathrm{d}}\right)\right\} \\
\geq \operatorname{tr}\left\{Q_{i_{0}} X_{i_{0}}\right\}+\alpha_{i_{0}}(0)+\mathrm{E}\left\{E_{\bar{R}_{0}}\left\{2 \theta_{i_{0}}{ }^{\prime}(0) \mathrm{x}_{0}\right\}\right\} \\
+\mathrm{E}\left\{\sum_{k=0}^{N-1} E_{\overline{R_{k}}}\left\{\left|\mathrm{D}_{\theta \mathrm{u}, \mathrm{m}(\mathrm{k})}(\mathrm{k}) \mathrm{E}_{\mathrm{m}(\mathrm{k})}\left(\theta_{c}^{-}(\mathrm{k}+1), \mathrm{k}\right)\right|_{T_{2, m(k)}}^{2}(k)\right\}\right\}+\mathrm{E}\left\{\bar{J}_{d}\left(\mathrm{r}_{\mathrm{d}}\right)\right\} \\
=\mathrm{J}_{\mathrm{dN}}\left(\mathrm{x}_{0}, \hat{u}_{d}^{*}, \mathrm{r}_{\mathrm{d}}\right)
\end{gathered}
$$

which concludes the proof of sufficiency.

Necessity:

Because of arbitrariness of the reference signal $r_{d}(\cdot)$, by considering the case of $r_{d}(\cdot) \equiv 0$, one can easily deduce the necessity for the solvability of the stochastic LQ optimal tracking problem [Costa et al. (2005); Fragoso (1989)]. Also notice that, in the proof of sufficiency, on the process of the evaluation of the performance index, by getting rid of the mixed terms of 
$\mathrm{r}_{\mathrm{d}}$ and $\mathrm{x}$, or $\theta_{m(k)}$ and $\mathrm{x}$, we necessarily obtain the form of the preview compensator dynamics. (Q.E.D.)

\section{Appendix 2. Proof of Orthogonal Property (10)}

In this appendix we give the proof of the orthogonal property (10).

We prove it by induction on $\mathrm{k}$.

For $\mathrm{k}=0$, since $\hat{x}_{e}(0)$ is deterministic,

$$
\mathrm{E}\left\{E_{\overline{R_{0}}}\left\{\mathrm{e}(0) \hat{x}_{e}{ }^{\prime}(0) 1_{\{\mathrm{m}(0)=\mathrm{i}\}}\right\}\right\}=\pi_{i}(0) \mathrm{E}\left\{E_{\overline{R_{0}}}\{\mathrm{e}(0)\}\right\} \hat{x}_{e}{ }^{\prime}(0)=\mathrm{O}
$$

We have already shown that, for each $\mathrm{k} \in[0, \mathrm{~N}]$,

$$
\mathrm{E}\left\{E_{\overline{R_{k}}}\left\{\mathrm{e}(\mathrm{k}) \bar{r}_{d, c}{ }^{\prime}(\mathrm{k}) 1_{\{\mathrm{m}(\mathrm{k})=\mathrm{i}\}}\right\}\right\}=\mathrm{O}
$$

in section 4. Suppose

$$
\mathrm{E}\left\{E_{\overline{R_{k}}}\left\{\mathrm{e}(\mathrm{k}) \hat{x}_{e}{ }^{\prime}(\mathrm{k}) 1_{\{\mathrm{m}(\mathrm{k})=\mathrm{i}\}}\right\}\right\}=\mathrm{O} .
$$

Then, since $\omega_{\mathrm{d}}(\mathrm{k})$ is zero mean, not correlated with $\hat{x}_{e}(\mathrm{k})$ and $\bar{r}_{d, c}(\mathrm{k})$ and independent of $\mathrm{m}(\mathrm{k})$, we have

$$
\begin{aligned}
& \mathrm{E}\left\{E_{\overline{R_{k+1}}}\left\{\mathrm{e}(\mathrm{k}+1) \hat{x}_{e}{ }^{\prime}(\mathrm{k}+1) 1_{\{\mathrm{m}(\mathrm{k}+1)=\mathrm{i}\}}\right\}\right\} \\
&=\sum_{i \in J(k)} p_{d, i j}[ {\left[\mathrm{A}_{\mathrm{d}, \mathrm{i}}+\mathrm{M}_{\mathrm{i}} \mathrm{C}_{\mathrm{i}}\right](\mathrm{k}) \mathrm{E}\left\{E_{\overline{R_{k}}}\left\{\mathrm{e}(\mathrm{k}) \hat{x}_{e}{ }^{\prime}(\mathrm{k}) 1_{\{\mathrm{m}(\mathrm{k})=\mathrm{i}\}}\right\}\right\}\left[\mathrm{A}_{\mathrm{d}, \mathrm{i}}+\mathrm{M}_{\mathrm{i}} \mathrm{C}_{2 \mathrm{~d}, \mathrm{i}}\right]^{\prime}(\mathrm{k}) } \\
&+ {\left[\mathrm{G}_{\mathrm{d}, \mathrm{i}}+\mathrm{M}_{\mathrm{i}} \mathrm{H}_{\mathrm{d}, \mathrm{i}}\right](\mathrm{k}) \mathrm{E}\left\{E_{\overline{R_{k}}}\left\{\omega_{\mathrm{d}}(\mathrm{k}) \hat{x}_{e}{ }^{\prime}(\mathrm{k}) 1_{\{\mathrm{m}(\mathrm{k})=\mathrm{i}\}}\right\}\right\}\left[\mathrm{A}_{\mathrm{d}, \mathrm{i}}+\mathrm{M}_{\mathrm{i}} \mathrm{C}_{2 \mathrm{~d}, \mathrm{i}}\right]^{\prime}(\mathrm{k}) } \\
&+\left[\mathrm{A}_{\mathrm{d}, \mathrm{i}}+\mathrm{M}_{\mathrm{i}} \mathrm{C}_{2 \mathrm{~d}, \mathrm{i}}\right](\mathrm{k}) \mathrm{E}\left\{E_{\overline{R_{k}}}\left\{\mathrm{e}(\mathrm{k}) \bar{u}_{d, c^{*}}(\mathrm{k}) 1_{\{\mathrm{m}(\mathrm{k})=\mathrm{i}\}}\right\}\right\} \mathrm{B}_{2 \mathrm{~d}, \mathrm{i}}{ }^{\prime}(\mathrm{k}) \\
&+\left[\mathrm{G}_{\mathrm{d}, \mathrm{i}}+\mathrm{M}_{\mathrm{i}} \mathrm{H}_{\mathrm{d}, \mathrm{i}}\right](\mathrm{k}) \mathrm{E}\left\{E_{\overline{R_{k}}}\left\{\omega_{\mathrm{d}}(\mathrm{k}) \bar{u}_{d, c^{*}}{ }^{\prime}(\mathrm{k}) 1_{\{\mathrm{m}(\mathrm{k})=\mathrm{i}\}}\right\}\right\} \mathrm{B}_{2 \mathrm{~d}, \mathrm{i}}{ }^{\prime}(\mathrm{k}) \\
&+\left[\mathrm{A}_{\mathrm{d}, \mathrm{i}}+\mathrm{M}_{\mathrm{i}} \mathrm{C}_{2 \mathrm{~d}, \mathrm{i}}\right](\mathrm{k}) \mathrm{E}\left\{E_{\overline{R_{k}}}\left\{\mathrm{e}(\mathrm{k}) \bar{r}_{d, c^{\prime}}(\mathrm{k}) 1_{\{\mathrm{m}(\mathrm{k})=\mathrm{i}\}}\right\}\right\} \mathrm{B}_{3 \mathrm{~d}, \mathrm{i}^{\prime}}(\mathrm{k}) \\
&+\left[\mathrm{G}_{\mathrm{d}, \mathrm{i}}+\mathrm{M}_{\mathrm{i}} \mathrm{H}_{\mathrm{d}, \mathrm{i}}\right](\mathrm{k}) \mathrm{E}\left\{E_{\overline{R_{k}}}\left\{\omega_{\mathrm{d}}(\mathrm{k}) \bar{r}_{d, c^{\prime}}(\mathrm{k}) 1_{\{\mathrm{m}(\mathrm{k})=\mathrm{i}\}}\right\}\right\} \mathrm{B}_{3 \mathrm{~d}, \mathrm{i}}{ }^{\prime}(\mathrm{k}) \\
&-\left[\mathrm{A}_{\mathrm{d}, \mathrm{i}}+\mathrm{M}_{\mathrm{i}} \mathrm{C}_{2 \mathrm{~d}, \mathrm{i}}\right](\mathrm{k}) \mathrm{E}\left\{E_{\overline{R_{k}}}\left\{\mathrm{e}(\mathrm{k}) \mathrm{y}^{\prime}(\mathrm{k}) 1_{\{\mathrm{m}(\mathrm{k})=\mathrm{i}\}}\right\}\right\} \mathrm{M}_{\mathrm{i}}{ }^{\prime}(\mathrm{k}) \\
&\left.-\left[\mathrm{G}_{\mathrm{d}, \mathrm{i}}+\mathrm{M}_{\mathrm{i}} \mathrm{H}_{\mathrm{d}, \mathrm{i}}\right](\mathrm{k}) \mathrm{E}\left\{E_{\overline{R_{k}}}\left\{\omega_{\mathrm{d}}(\mathrm{k}) \mathrm{y}^{\prime}(\mathrm{k}) 1_{\{\mathrm{m}(\mathrm{k})=\mathrm{i}\}}\right\}\right\} \mathrm{M}_{\mathrm{i}}{ }^{\prime}(\mathrm{k})\right] \\
&=\sum_{i \in J(k)} p_{d, i j}-\left[\mathrm{A}_{\mathrm{d}, \mathrm{i}}+\mathrm{M}_{\mathrm{i}} \mathrm{C}_{2 \mathrm{~d}, \mathrm{i}}\right](\mathrm{k}) \mathrm{E}\left\{E_{\overline{R_{k}}}\left\{\mathrm{e}(\mathrm{k}) \mathrm{y}^{\prime}(\mathrm{k}) 1_{\{\mathrm{m}(\mathrm{k})=\mathrm{i}\}}\right\}\right\} \mathrm{M}_{\mathrm{i}}{ }^{\prime}(\mathrm{k}) \\
&\left.-\left[\mathrm{G}_{\mathrm{d}, \mathrm{i}}+\mathrm{M}_{\mathrm{i}} \mathrm{H}_{\mathrm{d}, \mathrm{i}}\right](\mathrm{k}) \mathrm{E}\left\{E_{\overline{R_{k}}}\left\{\omega_{\mathrm{d}}(\mathrm{k}) \mathrm{y}^{\prime}(\mathrm{k}) 1_{\{\mathrm{m}(\mathrm{k})=\mathrm{i}\}}\right\}\right\} \mathrm{M}_{\mathrm{i}}{ }^{\prime}(\mathrm{k})\right]
\end{aligned}
$$


where $\bar{u}_{d, c^{*}}(\mathrm{k})=\mathrm{F}_{2, \mathrm{i}}(\mathrm{k}) \hat{x}_{e}(\mathrm{k}), \mathrm{i}=1, \cdots, \mathrm{M}$. Notice that

$$
\mathrm{y}(\mathrm{k})=\mathrm{C}_{2 \mathrm{~d}, \mathrm{~m}(\mathrm{k})}(\mathrm{k}) \mathrm{x}(\mathrm{k})+\mathrm{H}_{\mathrm{d}, \mathrm{m}(\mathrm{k})}(\mathrm{k}) \omega_{\mathrm{d}}(\mathrm{k})=\mathrm{C}_{2 \mathrm{~d}, \mathrm{~m}(\mathrm{k})}(\mathrm{k})\left(\mathrm{e}(\mathrm{k})+\hat{x}_{e}(\mathrm{k})\right)+\mathrm{H}_{\mathrm{d}, \mathrm{m}(\mathrm{k})}(\mathrm{k}) \omega_{\mathrm{d}}(\mathrm{k}) .
$$

Then, by induction on k, we obtain

$$
\begin{aligned}
\mathrm{E}\left\{E_{\overline{R_{k}}}\left\{\mathrm{e}(\mathrm{k}) \mathrm{y}^{\prime}(\mathrm{k}) 1_{\{\mathrm{m}(\mathrm{k})=\mathrm{i}\}}\right\}\right\}= & \mathrm{E}\left\{E_{\overline{R_{k}}}\left\{\mathrm{e}(\mathrm{k}) \mathrm{e}^{\prime}(\mathrm{k}) 1_{\{\mathrm{m}(\mathrm{k})=\mathrm{i}\}}\right\}\right\} \mathrm{C}_{2 \mathrm{~d}, \mathrm{i}^{\prime}(\mathrm{k})}+\mathrm{E}\left\{E_{\overline{R_{k}}}\left\{\mathrm{e}(\mathrm{k}) \hat{x}_{e}{ }^{\prime}(\mathrm{k}) 1_{\{\mathrm{m}(\mathrm{k})=\mathrm{i}\}}\right\}\right\} \mathrm{C}_{2 \mathrm{~d}, \mathrm{i}^{\prime}(\mathrm{k})} \\
& +\mathrm{E}\left\{E_{\overline{R_{k}}}\left\{\mathrm{e}(\mathrm{k}) \omega_{\mathrm{d}}{ }^{\prime}(\mathrm{k}) 1_{\{\mathrm{m}(\mathrm{k})=\mathrm{i}\}}\right\}\right\} \mathrm{H}_{\mathrm{d}, \mathrm{i}}{ }^{\prime}(\mathrm{k}) \\
= & \mathrm{Y}_{\mathrm{i}}(\mathrm{k}) \mathrm{C}_{2 \mathrm{~d}, \mathrm{i}^{\prime}}(\mathrm{k})
\end{aligned}
$$

We also obtain

$$
\begin{aligned}
& \mathrm{E}\left\{E_{\overline{R_{k}}}\left\{\omega_{\mathrm{d}}(\mathrm{k}) \mathrm{y}^{\prime}(\mathrm{k}) 1_{\{\mathrm{m}(\mathrm{k})=\mathrm{i}\}}\right\}\right\} \\
& =\mathrm{E}\left\{E_{\overline{R_{k}}}\left\{\omega_{\mathrm{d}}(\mathrm{k}) \mathrm{e}^{\prime}(\mathrm{k}) 1_{\{\mathrm{m}(\mathrm{k})=\mathrm{i}\}}\right\}\right\} \mathrm{C}_{2 \mathrm{~d}, \mathrm{i}^{\prime}}(\mathrm{k})+\mathrm{E}\left\{E_{\overline{R_{k}}}\left\{\omega_{\mathrm{d}}(\mathrm{k}) \hat{x}_{e}{ }^{\prime}(\mathrm{k}) 1_{\{\mathrm{m}(\mathrm{k})=\mathrm{i}\}}\right\}\right\} C_{2 \mathrm{~d}, \mathrm{i}^{\prime}}(\mathrm{k}) \\
& +\mathrm{E}\left\{E_{\overline{R_{k}}}\left\{\omega_{\mathrm{d}}(\mathrm{k}) \omega_{\mathrm{d}}{ }^{\prime}(\mathrm{k}) 1_{\{\mathrm{m}(\mathrm{k})=\mathrm{i}\}}\right\}\right\} \mathrm{H}_{\mathrm{d}, \mathrm{i}}{ }^{\prime}(\mathrm{k}) \\
& =\mathrm{E}\left\{\omega_{\mathrm{d}}(\mathrm{k}) \omega_{\mathrm{d}}{ }^{\prime}(\mathrm{k})\right\} \mathrm{P}\{\mathrm{m}(\mathrm{k})=\mathrm{i}\} \mathrm{H}_{\mathrm{d}, \mathrm{i}^{\prime}}(\mathrm{k})=\Pi_{\mathrm{i}}(\mathrm{k}) \mathrm{H}_{\mathrm{d}, \mathrm{i}^{\prime}}(\mathrm{k}) \text {. }
\end{aligned}
$$

Then considering the assumption A4 $\mathrm{G}_{\mathrm{d}, \mathrm{i}}(\mathrm{k}) \mathrm{H}_{\mathrm{d}, \mathrm{i}^{\prime}(\mathrm{k})}=\mathrm{O}, \mathrm{i}=1, \cdots, \mathrm{M}$, and

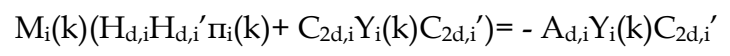

by (11), we finally obtain

$$
\begin{aligned}
\mathrm{E}\left\{E_{\overline{R_{k+1}}}\left\{\mathrm{e}(\mathrm{k}+1) \hat{x}_{e}{ }^{\prime}(\mathrm{k}+1) 1_{\{\mathrm{m}(\mathrm{k}+1)=\mathrm{i}\}}\right\}\right\} \\
\quad=\sum_{i \in J(k)} p_{d, i j}\left[-\left[\mathrm{A}_{\mathrm{d}, \mathrm{i}}+\mathrm{M}_{\mathrm{i}} \mathrm{C}_{2 \mathrm{~d}, \mathrm{i}}\right](\mathrm{k}) \mathrm{Y}_{\mathrm{i}}(\mathrm{k}) \mathrm{C}_{2 \mathrm{~d}, \mathrm{i}}{ }^{\prime}(\mathrm{k})-\left[\mathrm{G}_{\mathrm{d}, \mathrm{i}}+\mathrm{M}_{\mathrm{i}} \mathrm{H}_{\mathrm{d}, \mathrm{i}}\right](\mathrm{k}) \Pi_{\mathrm{i}}(\mathrm{k}) \mathrm{H}_{\mathrm{d}, \mathrm{i}}{ }^{\prime}(\mathrm{k})\right] \mathrm{M}_{\mathrm{i}}{ }^{\prime}(\mathrm{k}) \\
\quad=\sum_{i \in J(k)} p_{d, i j}\left[-\mathrm{A}_{\mathrm{d}, \mathrm{i}} \mathrm{Y}_{\mathrm{i}}(\mathrm{k}) \mathrm{C}_{2 \mathrm{~d}, \mathrm{i}}{ }^{\prime}(\mathrm{k})-\mathrm{M}_{\mathrm{i}}(\mathrm{k})\left(\mathrm{H}_{\mathrm{d}, \mathrm{i}} \mathrm{H}_{\mathrm{d}, \mathrm{i}}{ }^{\prime} \Pi_{\mathrm{i}}(\mathrm{k})+\mathrm{C}_{2 \mathrm{~d}, \mathrm{i}} \mathrm{Y}_{\mathrm{i}}(\mathrm{k}) \mathrm{C}_{2 \mathrm{~d}, \mathrm{i}}{ }^{\prime}\right)\right] \mathrm{M}_{\mathrm{i}}{ }^{\prime}(\mathrm{k}) \\
=\sum_{i \in J(k)} p_{d, i j}\left[-\mathrm{A}_{\mathrm{d}, \mathrm{i}} \mathrm{Y}_{\mathrm{i}}(\mathrm{k}) \mathrm{C}_{2 \mathrm{~d}, \mathrm{i}}{ }^{\prime}(\mathrm{k})+\mathrm{A}_{\mathrm{d}, \mathrm{i}} \mathrm{Y}_{\mathrm{i}}(\mathrm{k}) \mathrm{C}_{2 \mathrm{~d}, \mathrm{i}}{ }^{\prime}\right] \mathrm{M}_{\mathrm{i}}{ }^{\prime}(\mathrm{k}) \\
=0
\end{aligned}
$$

which concludes the proof. (Q.E.D.)

\section{References}

E. K. Boukas. (2006). Stochastic Switching Systems: Analysis and Design, Birkhauser, 0-81763782-6, Boston,

A. Cohen. \& U. Shaked. (1997). Linear Discrete-Time Hœ-Optimal Tracking with Preview. IEEE Trans. Automat. Contr., 42, 2, 270-276

O. L. V. Costa. \& E. F. Tuesta. (2003). Finite Horizon Quadratic Optimal Control and a Separation Principle for Markovian Jump Linear Systems. IEEE Trans. Automat. Contr., 48, 10, 1836-1842 
O. L. V. Costa.; M. D. Fragoso. \& R. P. Marques. (2005). Discrete-Time Markov Jump Linear Systems, Springer, 1-85233-761-3, London

V. Dragan. \& T. Morozan. (2004). The linear quadratic optimization problems for a class of linear stochastic systems with multiplicative white noise and Markovian jumping. IEEE Trans. Automat. Contr., 49, 5, 665-675

M. D. Fragoso. (1989). Discrete-Time Jump LQG Problem. Int. J. Systems Science, 20, 12, 25392545

M. D. Fragoso.; J. B. R. do Val . \& D. L. Pinto Junior. (1995). Jump Linear Hœ Control: the discrete-time case. Control-Theory and Advanced Technology, 10, 4, 1459-1474

E. Gershon.; D. J. N. Limebeer.; U. Shaked. \& I. Yaesh. (2004). Stochastic Ho Tracking with Preview for State-Multiplicative Systems. IEEE Trans. Automat. Contr., 49, 11, 20612068

E. Gershon.; U. Shaked. \& I. Yaesh. (2004). Hळ tracking of linear continuous-time systems with stochastic uncertainties and preview. Int. J. Robust and Nonlinear Control, 14, 7, 607-626

E. Gershon.; U. Shaked. \& I. Yaesh. (2005). Hœ Control and Estimation of State-Multiplicative Linear Systems, LNCIS 318, Springer, 1-85233-997-7, London

J.-W. Lee. \& P. P. Khargonekar. (2008). Optimal output regulation for discrete-time switched and Markovian jump linear systems, SIAM J. Control Optim., 47, 1, 40-72

M. Mariton. (1990). Jump Linear Systems in Automatic Control, Marcel Dekker, 0-8247-8200-3, New York

G. Nakura. (2008a). Noncausal Optimal Tracking for Linear Switched Systems. In: Hybrid Systems: Computation and Control: 11th International Workshop, HSCC 2008, St. Louis, MO, USA, April, 2008, Proceedings, LNCS 4981, M. Egerstedt. \& B. Mishra. (eds.), pp.372-385, Springer, 3-540-78928-6, Berlin, Heidelberg.

G. Nakura. (2008b). Ho Tracking with Preview for Linear Systems with Impulsive Effects State Feedback and Full Information Cases-. Proceedings of the 17th IFAC World Congress, TuA08.4 (CD-ROM), Seoul, Korea

G. Nakura. (2008c). Ho Tracking with Preview by Output Feedback for Linear Systems with Impulsive Effects. Proceedings of the 17th IFAC World Congress, TuA08.5 (CD-ROM), Seoul, Korea

G. Nakura. (2008d). Stochastic Optimal Tracking with Preview for Linear Continuous-Time Markovian Jump Systems. Proceedings of SICE Annual Conference 2008, 2A09-2 (CDROM), Chofu, Tokyo, Japan

G. Nakura. (2008e). Hळ Tracking with Preview for Linear Continuous-Time Markovian Jump Systems. Proceedings of SICE 8th Annual Conference on Control Systems, 073-2-1 (CD-ROM), Kyoto, Japan

G. Nakura. (2009). Stochastic Optimal Tracking with Preview for Linear Discrete-Time Markovian Jump Systems (Extended Abstract). In: Hybrid Systems: Computation and Control: 12th Conference, HSCC 2009, San Francisco, CA, USA, April, 2009, Proceedings, LNCS 5469, R. Majumdar. \& P. Tabuada. (Eds.), pp. 455-459, Springer, 3-642-006019, Berlin, Heidelberg 
G. Nakura. (2010). Stochastic Optimal Tracking with Preview by State Feedback for Linear Discrete-Time Markovian Jump Systems. International Journal of Innovative Computing, Information and Control (IJICIC), 6, 1, 15-27

Y. Sawada. (2008). Risk-sensitive tracking control of stochastic systems with preview action. International Journal of Innovative Computing, Information and Control (IJICIC), 4, 1, 189-198

U. Shaked. \& C. E. de Souza. (1995). Continuous-Time Tracking Problems in an Hळ Setting: A Game Theory Approach. IEEE Trans. Automat. Contr., 40, 5, 841-852

C. E. de Souza. \& M. D. Fragoso. (1993). Hœ Control for Linear Systems with Markovian Jumping Parameters. Control-Theory and Advanced Technology, 9, 2, 457-466

D. D. Sworder. (1969). Feedback Control of a Class of Linear Systems with Jump Parameters. IEEE Trans. Automat. Contr., AC-14, 1, 9-14

D. D. Sworder. (1972). Control of Jump Parameter Systems with Discontinuous State Trajectories. IEEE Trans. Automat. Contr., AC-17, 5, 740-741

K. Takaba. (2000). Robust servomechanism with preview action for polytopic uncertain systems. Int. J. Robust and Nonlinear Control, 10, 2, 101-111 


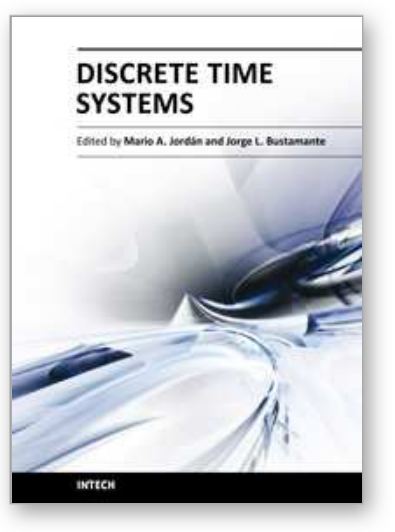

\author{
Discrete Time Systems \\ Edited by Dr. Mario Alberto Jord $\tilde{A}_{i n}$
}

ISBN 978-953-307-200-5

Hard cover, 526 pages

Publisher InTech

Published online 26, April, 2011

Published in print edition April, 2011

Discrete-Time Systems comprehend an important and broad research field. The consolidation of digital-based computational means in the present, pushes a technological tool into the field with a tremendous impact in areas like Control, Signal Processing, Communications, System Modelling and related Applications. This book attempts to give a scope in the wide area of Discrete-Time Systems. Their contents are grouped conveniently in sections according to significant areas, namely Filtering, Fixed and Adaptive Control Systems, Stability Problems and Miscellaneous Applications. We think that the contribution of the book enlarges the field of the Discrete-Time Systems with signification in the present state-of-the-art. Despite the vertiginous advance in the field, we also believe that the topics described here allow us also to look through some main tendencies in the next years in the research area.

\title{
How to reference
}

In order to correctly reference this scholarly work, feel free to copy and paste the following:

Gou Nakura (2011). Stochastic Optimal Tracking with Preview for Linear Discrete Time Markovian Jump Systems, Discrete Time Systems, Dr. Mario Alberto JordÃ in (Ed.), ISBN: 978-953-307-200-5, InTech, Available from: http://www.intechopen.com/books/discrete-time-systems/stochastic-optimal-tracking-withpreview-for-linear-discrete-time-markovian-jump-systems

\section{INTECH}

open science | open minds

\author{
InTech Europe \\ University Campus STeP Ri \\ Slavka Krautzeka 83/A \\ 51000 Rijeka, Croatia \\ Phone: +385 (51) 770447 \\ Fax: +385 (51) 686166 \\ www.intechopen.com
}

\author{
InTech China \\ Unit 405, Office Block, Hotel Equatorial Shanghai \\ No.65, Yan An Road (West), Shanghai, 200040, China \\ 中国上海市延安西路65号上海国际贵都大饭店办公楼 405 单元 \\ Phone: +86-21-62489820 \\ Fax: +86-21-62489821
}


(C) 2011 The Author(s). Licensee IntechOpen. This chapter is distributed under the terms of the Creative Commons Attribution-NonCommercialShareAlike-3.0 License, which permits use, distribution and reproduction for non-commercial purposes, provided the original is properly cited and derivative works building on this content are distributed under the same license. 